\title{
The BRST Cohomology of the NSR String: Vanishing and "No-Ghost" Theorems
}

\author{
José M. Figueroa-O’Farrill`, and Takashi Kimura ${ }^{\star}$ \\ Institute for Theoretical Physics, State University of New York at Stony Brook, \\ Stony Brook, NY 11794-3480, USA
}

\begin{abstract}
We investigate the BRST cohomology of the NSR string. We prove vanishing theorems for the full and relative (sub)complexes generalizing the work of Frenkel, Garland and Zuckerman for the bosonic string. Using these results we give simple proofs of the "no-ghost" theorems for both sectors.
\end{abstract}

\section{Introduction}

In [1] Frenkel, Garland, and Zuckerman computed the BRST cohomology of the open bosonic string after identifying it with a particular semi-infinite cohomology for which they had proven a key vanishing theorem; that is, that the BRST cohomology is zero except at zero ghost number. Their vanishing theorem did not just apply to that particular representation of the Virasoro algebra but to a large class of graded Lie algebras (including Kac-Moody algebras) and to a large class of their representations. In the present paper we extend their result to the representations of the super-Virasoro algebras appearing in the NSR string. The theorem admits some generalization which, lacking the string theoretic relevance of this special case, will be presented elsewhere. This way we can devote ourselves to the cases of current physical interest and therefore give a clear presentation of the method without needless generalizations.

The proof uses the algebraic machinery of spectral sequences. Since this lies somewhat outside the physicist's bag of tricks we thought it would be convenient to devote the next section to take a brief look at this powerful gadget. That section also serves to clarify the notation and the concepts concerning differential complexes that we use in this paper. We define the notion of a filtered complex and quote the main theorem concerning the spectral sequence associated to it. A very important special case of a filtered complex, and one for which we will find ample use, is the double complex. We will see that there are two canonical filtrations associated to a

\footnotetext{
* e-mail: (Bitnet) figueroa@sunysbnp (UUCP)....talcott!icus !noether!figueroa
}

$\star \star$ e-mail: (Bitnet) kimura@sunysbnp (UUCP)....talcott!icus!noether!kimura 
double complex and that the early terms of the spectral sequences associated to each of the filtrations are very easy to describe in terms of the two original differentials of the double complex. These theorems form the basis for the results in this paper. Since they are standard we refer the reader to the appropriate literature for the proofs.

The rest of the paper is organized as follows. Section 3 discusses in detail the vanishing theorem for the relative BRST subcomplex of the Neveu-Schwarz sector of the NSR string. In Sect. 4 we consider the Ramond sector. This is somewhat more complicated because of the existence of the superconformal ghosts' zero modes. In fact there has not appeared in the literature a unique treatment of these zero modes and thus we treat them in two different ways. The cohomologies turn out to be isomorphic although one of them does not admit a grading by ghost number. Therefore for this latter case the vanishing theorem does not make sense. In order to prove the vanishing theorems we use a basic result from the cohomology theory of Lie superalgebras. We have never seen a published proof of this theorem, although Fuks [2] hints that it is a straightforward generalization of the similar theorem for Lie algebras. We fill in the details in the first appendix for the special cases we need in this paper.

In Sect. 5 we use spectral sequences again to infer the vanishing theorem for the full BRST complex. This complex is half-integrally graded and what we show is that its cohomology is trivial except at ghost number $\pm \frac{1}{2}$. The spectral sequence used in this case is the one associated to one of the two canonical filtrations of a double complex. In Sect. 6 we prove the "no-ghost" theorems for the NSR string using the vanishing theorems proven earlier. Specifically what we prove is that the inherited norm on the BRST cohomology of the relative subcomplex (ignoring the ghosts' zero modes) is positive definite. This is a straightforward application of the method introduced in [1] and discussed in [3,4]. We will make constant use in this section of the contents of [4] to which we refer the reader. To be able to apply the results of [4] we need to show that we can find a positive-definite inner product for the Fock space where the BRST operator acts. The second appendix briefly describes this inner product.

\section{Spectral Sequences}

In this section we discuss briefly the basic notions of spectral sequences. For the proofs of the theorems we quote in this section, the reader is referred to the books by Lang [5], and Griffiths and Harris [6]. A more unified treatment of spectral sequences using Massey's concept of an "exact couple" can be found in the books by Bott and Tu [7], and Hilton and Stammbach [8]. A complete treatment with applications can be found in the book by MacLane [9].

A spectral sequence essentially allows us to approximate the cohomology of a complex by computing the cohomology of bigger and bigger chunks. By definition a spectral sequence is a sequence $\left\{\left(E_{r}, d_{r}\right)\right\}_{r=0,1, \ldots}$ of differential complexes ${ }^{1}$ where

\footnotetext{
${ }^{1}$ For our purposes a differential complex consists of a pair $(E, d)$ of a vector space $E$ and a linear map $d: E \rightarrow E$, called the differential, obeying $d^{2}=0$
} 
$E_{r+1}$ is the cohomology space of the preceding complex $\left(E_{r}, d_{r}\right)$. That is,

$$
E_{r+1}=\frac{\operatorname{ker} d_{r}}{\operatorname{im} d_{r}} .
$$

In many cases of interest one has that for $r>R, E_{r}=E_{r+1}=\cdots=E_{\infty}$. In this case one says that the spectral sequence converges to $E_{\infty}$ and one writes $\left(E_{r}\right) \Rightarrow E_{\infty}$.

The following is the typical use to which spectral sequences are put to in practice. One generally finds oneself in the situation where the cohomology $H$ of a certain complex is to be computed. Then one shows (usually by very general arguments) that there exists a spectral sequence converging to $H$, whose early (first or second) terms are easily computable. Thus one begins to approximate $H$. It may be that after the first or second term the differentials $\left\{d_{r}\right\}$ are identically zero. Then that term is already isomorphic to the limit term $E_{\infty}$, in which case the spectral sequence is said to degenerate at the $E_{1}$ or $E_{2}$ terms. In that case we have reduced the computation of $H$ to the computation of the cohomology of a much simpler complex. We will see an example of this application in Sects. 4 and 5.

Sometimes however we are not so lucky and the spectral sequence does not degenerate early, yet it still provides us with a lot of useful information. In particular it can be used to obtain vanishing theorems. This will be the use for them in Sects. 3 and 4. It is worth elaborating on this point.

Usually complexes come equipped with a grading under which the differential acquires a well-defined degree. Let us suppose for definiteness that $E=\bigoplus_{n} E^{n}$ and $d: E^{n} \rightarrow E^{n+1}$. Then the cohomology is also graded: $H=\bigoplus_{n} H^{n}$, in the obvious manner. In the cases that will occupy our attention the spectral sequence converging to $H$ will respect the grading, and therefore we will have convergence in each dimension: $\left(E_{r}^{n}\right) \Rightarrow H^{n}$ for all $n$. From the definition of the spectral sequence we notice that $E_{r+1}^{n}$ is a subquotient of $E_{r}^{n}$ and hence if for any $r$ we have a vanishing of cohomology, say, $E_{r}^{n}=0$ for some $n$, then the vanishing will persist and $H^{n}=0$. This propagation of vanishing of cohomology is, in a nutshell, the essence of the vanishing theorem of Frenkel, Garland, and Zuckerman and of our generalization. Indeed we find that there is a vanishing theorem for the $E_{1}$ term in a spectral sequence converging to the (reduced) BRST cohomology of the NSR string.

We now describe in some detail the spectral sequences arising in this paper. They are all special cases of the spectral sequence which arises from a filtered complex, so we start by considering these.

Let $(C, d)$ be a differential complex. By a filtration of $C$ we mean a sequence (not necessarily finite) of subspaces $F C=\left\{F^{p} C\right\}$ indexed by an integer ${ }^{2} p$-called the filtration degree - such that, for all $p, F^{p} C \supseteq F^{p+1} C$ and such that $\cup_{p} F^{p} C=C$. We will deal exclusively with filtrations which are bounded: that is, there exist $p_{0}$ and $p_{1}$ such that

$$
F^{p} C=\left\{\begin{array}{ll}
C & \text { for } p \leqq p_{0} \\
0 & \text { for } p \geqq p_{1}
\end{array} .\right.
$$

If the differential respects the filtration, that is, $d F^{p} C \subseteq F^{p} C$, then $(F C, d)$ is called a filtered differential complex.

\footnotetext{
${ }^{2}$ This is only for definiteness. In this paper, for instance, we will use both integral and half integral filtrations
} 
Let $F C$ be a bounded filtered complex. Then each $F^{p} C$ is, in its own right, a complex under $d$ and, therefore, its cohomology can be defined. The inclusion $F^{p} C \subseteq C$ induces a map in cohomology $H\left(F^{p} C\right) \rightarrow H(C)$ which, however, is generally not injective. To understand this notice that a cocycle in $F^{p} C$ may be the differential of a cochain which does not belong to $F^{p} C$ but to $F^{p-1} C$. Therefore the cohomology class it defines may not be trivial in $H\left(F^{p} C\right)$ but it may be in $H(C)$. Let us denote by $F^{p} H(C) \subseteq H(C)$ the image of $H\left(F^{p} C\right)$ under the aforementioned map. It is easy to verify that $F H(C)$ defines a filtration of $H(C)$ which is bounded if $F C$ is.

To every filtered vector space $F C$ we can associate a graded vector space Gr $C=\bigoplus_{p} \mathrm{Gr}^{p} C$ where

$$
\mathrm{Gr}^{p} C \equiv F^{p} C / F^{p+1} C
$$

It is easy to see that as vector spaces $C$ and $\mathrm{Gr} C$ are isomorphic; although, since $C$ is not necessarily graded, this isomorphism does not extend to an isomorphism of graded spaces.

If $(F C, d)$ is a filtered differential complex then the associated graded space $\mathrm{Gr} C$ is also a complex whose differential is induced by $d$. Notice that if $F C$ is bounded then $\operatorname{Gr} C$ is actually finite. Since $d$ respects the filtration, upon passage to the quotient we obtain a map, also called $d$, which maps $d: \mathrm{Gr}^{p} C \rightarrow \mathrm{Gr}^{p} C$, whose cohomology is denoted by $H(\mathrm{Gr} C)$. Notice that whereas $\operatorname{Gr} C$ is graded, the differential has degree zero. This cohomology is usually easier to calculate than $H(C)$ or $H(F C)$; the reason being that the differential in the associated graded complex is usually a simpler operator. It may be that parts of $d$ have positive filtration degree, mapping $F^{p} C \rightarrow F^{p+1} C$, in which case this is already zero in $\mathrm{Gr}^{p} C$.

The spectral sequence of a filtered complex relates the two spaces $\operatorname{Gr} H(C)$ and $H(\mathrm{Gr} C)$. In fact we have the following

Theorem. Let FC be a bounded filtered complex and $\mathrm{Gr} C$ its associated graded complex. Then there exists a spectral sequence $\left\{\left(E_{r}, d_{r}\right)\right\}$ of graded spaces

with

$$
E_{r}=\bigoplus_{p} E_{r}^{p}
$$

and such that

$$
d_{r}: E_{r}^{p} \rightarrow E_{r}^{p+r}
$$

$$
E_{0}^{p} \cong \mathrm{Gr}^{p} C, \quad E_{1}^{p} \cong H\left(\mathrm{Gr}^{p} C\right), \quad \text { and } \quad E_{\infty}^{p} \cong \mathrm{Gr}^{p} H(C) .
$$

Moreover the spectral sequence converges finitely to the limit term.

Now suppose that $C$ is a graded complex $C=\bigoplus_{n} C^{n}$ - where $n$ will be called the dimension - such that the differential has degree 1 ,

$$
d: C^{n} \rightarrow C^{n+1}
$$

and let $F C$ be a filtration of $C$. In this case we can grade the filtration as follows: $F^{p} C=\bigoplus_{n} F^{p} C^{n}$, where $F^{p} C^{n}=F^{p} C \cap C^{n}$. The associated graded complex is now bigraded as follows: $\operatorname{Gr} C=\bigoplus_{p, n} \mathrm{Gr}^{p} C^{n}$ with the obvious definition for $\mathrm{Gr}^{p} C^{n}$. Supposing that the filtration is bounded in each dimension we get a slightly modified version of the previous theorem: 
Theorem. Let $C$ be a graded complex, FC be a filtration which is bounded in each dimension and $\mathrm{Gr} C$ its associated graded complex. Then there exists a spectral sequence $\left\{\left(E_{r}, d_{r}\right)\right\}$ of bigraded spaces

with

$$
E_{r}=\bigoplus_{p, q} E_{r}^{p, q}
$$

and such that

$$
d_{r}: E_{r}^{p, q} \rightarrow E_{r}^{p+r, q-r+1}
$$

$$
E_{0}^{p, q} \cong \mathrm{Gr}^{p} C^{p+q}, \quad E_{1}^{p, q} \cong H^{p+q}\left(\mathrm{Gr}^{p} C\right), \quad \text { and } \quad E_{\infty}^{p, q} \cong \mathrm{Gr}^{p} H^{p+q}(C) .
$$

Moreover the spectral sequence converges finitely to the limit term.

Very important special cases of a filtered complex arise from a double complex. A double complex is a bigraded vector space $\mathbf{K}=\bigoplus_{p, q} \mathbf{K}^{p, q}$ - where, for definiteness, we take $p, q$ integral, although this is not essential - and two differentials

$$
D^{\prime}: \mathbf{K}^{p, q} \rightarrow \mathbf{K}^{p+1, q}, \quad D^{\prime \prime}: \mathbf{K}^{p, q} \rightarrow \mathbf{K}^{p, q+1}
$$

which anticommute. It is often convenient to represent the double complex pictorially as follows

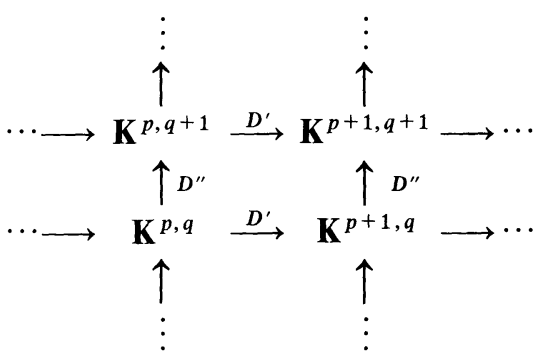

Hence we shall refer to $D^{\prime}$ and $D^{\prime \prime}$ as the horizontal and vertical differentials, respectively.

Defining the total degree of vectors in $\mathbf{K}^{p, q}$ as $p+q$ we may form a graded complex called the total complex and denoted by $\operatorname{Tot} \mathbf{K}=\bigoplus_{n} \operatorname{Tot}^{n} \mathbf{K}$, where

$$
\operatorname{Tot}^{n} \mathbf{K}=\bigoplus_{p+q=n} \mathbf{K}^{p, q}
$$

The differential in the total complex is $D=D^{\prime}+D^{\prime \prime}$ and is called the total differential. Since the total differential has total degree 1,

$$
D: \operatorname{Tot}^{n} \mathbf{K} \rightarrow \operatorname{Tot}^{n+1} \mathbf{K},
$$

(Tot $\mathbf{K}, D$ ) becomes a graded complex. We shall deal exclusively with double complexes which satisfy a mild finiteness condition: for each $n$ there are only a finite number of non-zero $\mathbf{K}^{p, q}$ with $p+q=n$.

There are two natural filtrations associated to the graded complex Tot $\mathbf{K}$. Define

$$
{ }^{\prime} F^{p} \text { Tot } \mathbf{K}=\bigoplus_{q} \underset{i \geqq p}{\bigoplus} \mathbf{K}^{i, q} \quad \text { and } \quad " F^{q} \text { Tot } \mathbf{K}=\bigoplus_{p} \underset{j \geqq q}{\bigoplus} \mathbf{K}^{p, j} .
$$


Fix $n$ and define

$$
{ }^{\prime} F^{p} \operatorname{Tot}^{n} \mathbf{K}=\bigoplus_{i \geqq p} \mathbf{K}^{i, n-i} \text { and } \quad " F^{q} \operatorname{Tot}^{n} \mathbf{K}=\underset{j \geqq q}{\bigoplus} \mathbf{K}^{n-j, j} .
$$

The finiteness condition for the double complex imply that the above filtrations are bounded for each $n$. Therefore, for each $n$, there exist $p_{0}, p_{1}, q_{0}$, and $q_{1}-$ which depend on $n$ - such that

and

$$
{ }^{\prime} F^{p} \operatorname{Tot}^{n} \mathbf{K}=\left\{\begin{array}{ll}
\operatorname{Tot}^{n} \mathbf{K} & \text { for } p \leqq p_{0} \\
0 & \text { for } p \geqq p_{1}
\end{array},\right.
$$

$$
{ }^{\prime} F^{q} \operatorname{Tot}^{n} \mathbf{K}=\left\{\begin{array}{ll}
\operatorname{Tot}^{n} \mathbf{K} & \text { for } q \leqq q_{0} \\
0 & \text { for } q \geqq q_{1}
\end{array} .\right.
$$

By the previous theorem there is a spectral sequence associated to each of the filtrations defined above which converges finitely to the cohomology of the total complex (Tot $\mathbf{K}, D$ ). What makes this example so important is that the earliest terms in the spectral sequence are easily described in terms of the original data $\left(\mathbf{K}, D^{\prime}, D^{\prime \prime}\right)$. In fact one finds for the horizontal filtration:

Theorem'. Associated to the filtration 'FTot $\mathbf{K}$ there exists a spectral sequence $\left\{\left({ }^{\prime} E_{r}, d_{r}\right)\right\}_{r=0,1, \ldots}$ of bigraded vector spaces

such that

$$
' E_{r}=\bigoplus_{p, q}^{\prime} E_{r}^{p, q} \quad \text { with } \quad d_{r}: E_{r}^{p, q} \rightarrow{ }^{\prime} E_{r}^{p+r, q-r+1}
$$

and

$$
\begin{aligned}
& { }^{\prime} E_{0}^{p, q} \cong \mathbf{K}^{p, q} \\
& { }^{\prime} E_{1}^{p, q} \cong{ }^{\prime \prime} H^{p, q}(\mathbf{K}), \\
& { }^{\prime} E_{2}^{p, q} \cong{ }^{\prime} H^{p}\left(" H^{q}(\mathbf{K})\right),
\end{aligned}
$$

$$
{ }^{\prime} E_{\infty}^{p, q} \cong \mathrm{Gr}^{p} H^{p+q}(\operatorname{Tot} \mathbf{K})
$$

We must explain the notation. In this theorem, by " $H^{p, q}(\mathbf{K})$ we mean the $q^{\text {th }}$ cohomology of the complex (which appears vertically in the double complex)

$$
\cdots \rightarrow \mathbf{K}^{p, q} \stackrel{D^{\prime \prime}}{\rightarrow} \mathbf{K}^{p, q+1} \rightarrow \cdots ;
$$

whereas by ' $H^{p}\left(" H^{q}(\mathbf{K})\right)$ we mean the $q^{\text {th }}$ cohomology of the complex

$$
\cdots \rightarrow " H^{p, q}(\mathbf{K}) \stackrel{D^{\prime}}{\rightarrow} " H^{p+1, q}(\mathbf{K}) \rightarrow \cdots
$$

which is well defined since $D^{\prime}$ and $D^{\prime \prime}$ anticommute.

Similarly for the vertical filtration we have the following

Theorem". Associated to the filtration "FTot $\mathbf{K}$ there exists a spectral sequence $\left\{\left({ }^{\prime \prime} E_{r}, d_{r}\right)\right\}_{r=0,1, \ldots}$ of bigraded vector spaces

$$
" E_{r}=\bigoplus_{p, q} " E_{r}^{q, p} \quad \text { with } \quad d_{r}: " E_{r}^{q, p} \rightarrow " E_{r}^{q+r, p-r+1}
$$


such that

$$
{ }^{\prime \prime} E_{0}^{q, p} \cong \mathbf{K}^{p, q}, \quad{ }^{\prime \prime} E_{1}^{q, p} \cong{ }^{\prime} H^{p, q}(\mathbf{K}), \quad{ }^{\prime} E_{2}^{q, p} \cong{ }^{\prime \prime} H^{q}\left({ }^{\prime} H^{p}(\mathbf{K})\right),
$$

and

$$
{ }^{\prime \prime} E_{\infty}^{q, p} \cong \mathrm{Gr}^{q} H^{p+q}(\operatorname{Tot} \mathbf{K}) .
$$

Similar notational remarks to the ones preceding this last theorem apply here as well.

\section{The Neveu-Schwarz Sector}

In this section we define the relative subcomplexes for the super-Virasoro algebra appearing in the Neveu-Schwarz sector of the NSR string and we prove a vanishing theorem for its cohomology. We use a Poincaré duality result proven in [4] for the BRST cohomology of a Fock space possessing a positive definite inner product. In Appendix B we construct this inner product for the Fock space of the NSR string.

Let $\mathscr{N} \mathscr{S}$ denote the centrally extended complexified super-Virasoro algebra appearing in the Neveu-Schwarz sector of the NSR string. This is a Lie superalgebra whose even part is the Virasoro algebra $\mathscr{L}=\bigoplus_{n} \mathscr{L}_{n}$. Each $\mathscr{L}_{n}$ is spanned by $l_{n}$ for $n$ different from zero and $\mathscr{L}_{0}$ is spanned by $l_{0}$ and $c$. The Virasoro algebra is defined by

$$
\left[l_{m}, l_{n}\right]=(m-n) l_{m+n}+\frac{c}{12} m\left(m^{2}-1\right) \delta_{m,-n},
$$

and by the fact that $c$ is central. Also let us define $\mathscr{L}_{ \pm}=\bigoplus_{ \pm n>0} \mathscr{L}_{n}$.

The odd part of $\mathscr{N} \mathscr{S}$ is graded according to $\mathscr{G}=\bigoplus_{r \in \mathbb{Z}+\frac{1}{2}} \mathscr{G}_{r}$, where $\mathscr{G}_{r}$ is spanned by $g_{r}$. These generators obey

and

$$
\left\{g_{r}, g_{s}\right\}=2 l_{r+s}+\frac{c}{3}\left(r^{2}-\frac{1}{4}\right) \delta_{r,-s}
$$

$$
\left[l_{n}, g_{r}\right]=\left(\frac{n}{2}-r\right) g_{r+n}
$$

Supplementing these relations by the assertion that $c$ is central fully defines the super-Virasoro algebra in this sector. Again we define $\mathscr{N} \mathscr{S}_{ \pm}=\mathscr{L}_{ \pm} \oplus \bigoplus_{ \pm r>0} \mathscr{G}_{r}$.

As is well known the ghost Fock space of the Neveu-Schwarz sector carries a representation of $\mathscr{N} \mathscr{S}$ where $c \mapsto-15$ Id and $l_{n} \mapsto L_{n}^{\text {ghost }}, g_{r} \mapsto G_{r}^{\text {ghost }}$. The Fock space of the string oscillators also carries a representation of $\mathscr{N} \mathscr{S}$ with the opposite central charge (in the critical dimension) and where $l_{n} \mapsto L_{n}^{\text {matter }}$ and $g_{r} \mapsto G_{r}^{\text {matter }}$. Let us denote by $L_{n}$ and $\mathrm{G}_{r}$ the operators representing $l_{n}$ and $g_{r}$ respectively in the full Fock space (including ghosts). The formulas for these generators are standard and can be found for instance in [10].

It was proven by Brower and Friedman [11] that this representation is fully reducible. That is, it can be written as an infinite direct sum of Verma modules whose highest weight vectors are obtained by repeated application of the creation 
operators in the full spectrum-generating algebra. ${ }^{3}$ Since the BRST operator commutes with the $\left\{L_{n}\right\}$ and the $\left\{G_{r}\right\}$ it respects this decomposition, and hence we may restrict our attention to one such Verma module at a time when computing the BRST cohomology. Let $\mathscr{M}$ denote one such Verma module. Following [1] we denote the BRST (or semi-infinite) cohomology ${ }^{4}$ of the $\mathscr{N} \mathscr{S}$ superalgebra with coefficients in $\mathscr{M}$ by $H_{\infty}(\mathscr{N} \mathscr{S} ; \mathscr{M})$. This is the cohomology of the BRST operator $Q$ acting on the graded complex $C_{\infty}(\mathscr{N} \mathscr{S} ; \mathscr{M})=\bigoplus_{n} C_{\infty}^{n}(\mathscr{N} \mathscr{S} ; \mathscr{M})$, where

$$
C_{\infty}^{n}(\mathscr{N} \mathscr{S} ; \mathscr{M})=C_{\infty}^{n}(\mathscr{N} \mathscr{S}) \otimes \mathscr{M},
$$

where $C_{\infty}^{n}(\mathscr{N} \mathscr{S})$ is the subspace of the ghost Fock space at ghost number $n$.

Let us define a subcomplex of $C_{\infty}(\mathscr{N} \mathscr{S} ; \mathscr{M})$ - called the subcomplex relative to $\mathscr{L}_{0}-$ by

$$
C_{\infty}\left(\mathscr{N} \mathscr{S}, \mathscr{L}_{0} ; \mathscr{M}\right)=\left\{\omega \in C_{\infty}(\mathscr{N} \mathscr{S} ; \mathscr{M}) \mid L_{0} \omega=b_{0} \omega=0\right\}
$$

For the sake of notation let us abbreviate $C_{\infty}\left(\mathscr{N} \mathscr{S}, \mathscr{L}_{0} ; \mathscr{M}\right)$ with $C_{\infty}$. Notice that $C_{\infty}$ is finite dimensional. From the identity $\left\{Q, b_{0}\right\}=L_{0}$ we notice that this indeed defines a subcomplex. That is, $Q C_{\infty} \subseteq C_{\infty}$. We denote its cohomology by $H_{\infty}\left(\mathcal{N} \mathscr{S}, \mathscr{L}_{0} ; \mathscr{M}\right)$.

Let

$$
|i, j, k, l, m, q\rangle=\prod_{r \geqq \frac{1}{2}} \gamma_{-r}^{i_{r}} \prod_{r \geqq \frac{1}{2}} \beta_{-r}^{j_{r}} \prod_{n>0} c_{-n}^{k_{n}} \prod_{n>0} b_{-n}^{l_{n}}|0\rangle \otimes \prod_{r \geqq \frac{1}{2}} G_{-r}^{q_{r}} \prod_{n>0} L_{-n}^{m_{n}}|p\rangle
$$

denote a vector in $C_{\infty}$ with $|p\rangle$ a highest weight vector of momentum $p$ such that

$$
\frac{1}{2}\left(p^{2}-1\right)=-\left(\sum_{n}\left(k_{n}+l_{n}+m_{n}\right) n+\sum_{r}\left(i_{r}+j_{r}+q_{r}\right) r\right)=-\frac{N}{2},
$$

for some non-negative integer $N$. Define the filtration degree as

$$
\operatorname{fdeg}|i, j, k, l, m, q\rangle=\sum_{n}\left(k_{n}-l_{n}-m_{n}\right) n+\sum_{r}\left(i_{r}-j_{r}-q_{r}\right) r
$$

This allows us to define a half-integral filtration of $C_{\infty}$ by

$$
F^{p} C_{\infty}=\left\{\omega \in C_{\infty} \mid \mathrm{fdeg} \omega \geqq p\right\} .
$$

First of all notice that $F^{p} C_{\infty} \supseteq F^{p+\frac{1}{2}} C_{\infty}$ and that the filtration is bounded. Finally we must check that this indeed defines a filtered complex, that is, $Q F^{p} C_{\infty} \subseteq F^{p} C_{\infty}$. This is done by examining the filtration degree of the homogeneous terms in $Q$ and making sure they are all non-negative. From (3.8) we can read off the filtration degree of all the oscillators which make up $Q$ and we find them to be the following:

\footnotetext{
${ }^{3}$ Strictly speaking, this is not true for the case of zero center of mass momentum. In this case the highest weight vector is also annihilated by $G_{-\frac{1}{2}}^{\text {matter }}$, and hence does not generate a Verma module. For this case the theorem in Appendix $A$ does not hold and neither does our proof of the vanishing theorem. Here, however, the BRST cohomology is easy to compute explicitly

${ }^{4}$ To be precise, this is the cohomology relative to the center. In other words, from now on $\mathscr{N} \mathscr{S}$ denotes the unextended Neveu-Schwarz algebra
} 


\begin{tabular}{lc}
\hline Operator & Filtration degree \\
\hline$c_{n}$ & $|n|$ \\
$b_{n}$ & $-|n|$ \\
$\gamma_{r}$ & $|r|$ \\
$\beta_{r}$ & $-|r|$ \\
$L_{n}^{\text {matter }}$ & $n$ \\
$G_{r}^{\text {matter }}$ & $r$ \\
\hline
\end{tabular}

Therefore it is trivial to verify that all terms in $Q$ have zero filtration degree except for the terms $L_{n}^{\text {matter }} c_{-n}$ for $n>0$ which have filtration degree $2 n$; the terms $G_{r}^{\text {matter }} \gamma_{-r}$ for $r>0$ which have filtration degrees $2 r$; the terms $c_{m} c_{n} b_{-(m+n)}$ for $\operatorname{sign}(m) \neq \operatorname{sign}(n)$ which have filtration degree $|m|+|n|-|m+n|$; the terms $\gamma_{r} \gamma_{s} b_{-(r+s)}$ for $\operatorname{sign}(r) \neq \operatorname{sign}(s)$ which have filtration degree $|r|+|s|-|r+s|$; and finally the terms $\gamma_{r} c_{n} \beta_{-(r+n)}$ for $\operatorname{sign}(r) \neq \operatorname{sign}(n)$ which have filtration degree $|r|+|n|-|r+n|$. Hence all terms have non-negative filtration degrees and $\left\{F^{p} C_{\infty}\right\}$ indeed defines a bounded filtered complex.

By the theorem in Sect. 2 there exists a spectral sequence converging finitely to $H_{\infty}\left(\mathscr{N} \mathscr{S}, \mathscr{L}_{0} ; \mathscr{M}\right)$ whose $E_{1}$ term is the cohomology of the associated graded complex Gr $C_{\infty}=\bigoplus_{p} \mathrm{Gr}^{p} C_{\infty}$, where $\mathrm{Gr}^{p} C_{\infty}=F^{p} C_{\infty} / F^{p+\frac{1}{2}} C_{\infty}$. The differential in this complex is precisely the part of $Q$ with zero filtration degree since the terms with positive filtration degree will automatically map to zero in $\operatorname{Gr} C_{\infty}$. By the above discussion the induced differential can be seen to be the differential on the complex

$$
C^{L_{0}}=\left(C\left(\mathscr{N} \mathscr{S}_{+}\right) \otimes C_{\infty}\left(\mathscr{N} \mathscr{S}_{-} ; \mathscr{M}\right)\right)^{L_{0}},
$$

where $C\left(\mathscr{N} \mathscr{S}_{+}\right)$denotes the Lie superalgebra cochains ${ }^{5}$ of $\mathscr{N} \mathscr{S}_{+}$with coefficients in the trivial representation, ()$^{L_{0}}$ denotes the $L_{0}$ invariant subspace and $\mathscr{M}$ is to be thought of as a respresentation of only $\mathscr{N} \mathscr{S}_{-}$. We remark that this particular expression makes it very easy to keep track of ghosts and antighosts separately. In fact, the subspace of $C^{L_{0}}$ with $c$ ghost and $b$ antighosts is just

$$
\left(C^{L_{0}}\right)^{b, c}=\left(C^{c}\left(\mathscr{N} \mathscr{S}_{+}\right) \otimes C_{\infty}^{b}\left(\mathscr{N} \mathscr{S}_{-} ; \mathscr{M}\right)\right)^{L_{0}} .
$$

We now compute this cohomology. Since $L_{0}$ is diagonalizable in $C$,

$$
C=C^{L_{0}} \oplus L_{0}(C) \text {, }
$$

where $L_{0}(C)$ denotes the image of $C$ under $L_{0}$. Since $L_{0}$ commutes with $Q$ we deduce that

and

$$
Q C^{L_{0}} \subseteq C^{L_{0}}
$$

$$
Q L_{0}(C) \subseteq L_{0}(C)
$$

Now suppose that $\omega$ is an $L_{0}$ invariant cocycle. If $\omega=Q \phi$ then we can choose $\phi$ to be $L_{0}$ invariant as well. To see this notice that if $\phi$ is not $L_{0}$ invariant already then by

\footnotetext{
${ }^{5}$ Strictly speaking we mean here cochains of finite support. That is, super-symmetric linear functionals of finite rank. They correspond to polynomials in the ghost creation operators
} 
(3.12) $\phi=\phi_{0}+\psi$, where $\phi_{0} \in C^{L_{0}}$ and $\psi \in L_{0}(C)$. Then $\omega=Q \phi=Q \phi_{0}+Q \psi$. By (3.13) and (3.14) $Q \psi=0$ and therefore $\omega=Q \phi_{0}$. Hence we have proven the inclusion

$$
H\left(C^{L_{0}}\right) \subseteq H(C)^{L_{0}} .
$$

The reverse inclusion is easier. If $[\omega] \in H(C)^{L_{0}}$ then $L_{0} \omega=\left(Q b_{0}+b_{0} Q\right) \omega=0$ since $Q \omega=0$ and $b_{0} \omega=0$. Therefore $\omega \in C^{L_{0}}$ defines a class in $H\left(C^{L_{0}}\right)$ which, if trivial, is trivial also in $H(C)^{L_{0}}$. Therefore we conclude that

$$
H(C)^{L_{0}} \cong H\left(C^{L_{0}}\right)
$$

But by the Künneth formula

$$
H(C) \cong H\left(\mathscr{N} \mathscr{S}_{+}\right) \otimes H_{\infty}\left(\mathscr{N} \mathscr{S}_{-} ; \mathscr{M}\right) ;
$$

whence, keeping track of ghosts and antighosts separately, the $E_{1}$ term in the spectral sequence is

$$
E_{1}^{b, c}=\left(H^{c}\left(\mathscr{N} \mathscr{S}_{+}\right) \otimes H_{\infty}^{b}\left(\mathscr{N} \mathscr{S}_{-} ; \mathscr{M}\right)\right)^{L_{0}} .
$$

In Appendix A we prove that $H_{\infty}^{b}\left(\mathscr{N} \mathscr{S}_{-} ; \mathscr{M}\right)=0$ for $b \neq 0$ and $H_{\infty}^{0}\left(\mathscr{N} \mathscr{S}_{-} ; \mathscr{M}\right) \cong \mathbb{C}$. Thus,

$$
\begin{gathered}
E_{1}^{m}=\bigoplus_{c-b=m} E_{1}^{b, c}=E_{1}^{0, m}=\left(H^{m}\left(\mathcal{N} \mathscr{S}_{+}\right)\right)^{L_{0}} \\
\therefore E_{1}^{m}=0 \text { for } m<0 .
\end{gathered}
$$

But $\left(E_{r}^{m}\right) \Rightarrow H_{\infty}^{m}\left(\mathcal{N} \mathscr{S}, \mathscr{L}_{0} ; \mathscr{M}\right)$, thus $H_{\infty}^{m}\left(\mathscr{N} \mathscr{S}, \mathscr{L}_{0} ; \mathscr{M}\right)=0$ for $m<0$. Taking into account all the Verma modules $\mathscr{M}$ we find that $H_{\infty}^{m}\left(\mathscr{N} \mathscr{S}, \mathscr{L}_{0} ; \mathscr{H}\right)=0$ for $m<0$, where $\mathscr{H}$ is the full Fock space (including ghosts) of the Neveu-Schwarz string.

Now in Appendix B we show that there exists a positive definite inner product in $\mathscr{H}$. This and the obvious fact that $\mathscr{H}$ breaks up into finite dimensional subspaces stabilized by $Q$ allow us to use the Poincare duality theorem proven in [4]:

$$
H_{\infty}^{m}\left(\mathscr{N} \mathscr{S}, \mathscr{L}_{0} ; \mathscr{H}\right) \cong H_{\infty}^{-m}\left(\mathscr{N} \mathscr{S}, \mathscr{L}_{0} ; \mathscr{H}\right)
$$

which gives the vanishing theorem for the relative subcomplex

$$
H_{\infty \neq 0}^{m}\left(\mathcal{N} \mathscr{S}, \mathscr{L}_{0} ; \mathscr{H}\right)=0 .
$$

In the Sect. 5 we will prove that this induces a vanishing theorem in the full complex $H_{\infty}(\mathscr{N} \mathscr{S} ; \mathscr{H})$ as well.

\section{The Ramond Sector}

Let $\mathscr{R}$ denote the centrally extended complexified super-Virasoro algebra appearing in the Ramond sector of the NSR string. This algebra is very similar to the Neveu-Schwarz algebra except that the odd part $\mathscr{F}=\bigoplus_{n \in \mathbb{Z}} \mathscr{F}_{n}$ is integrally graded, where $\mathscr{F}_{n}$ is spanned by $f_{n}$. The even subalgebra is still given by (3.1). The rest of the algebra obeys

$$
\left\{f_{m}, f_{n}\right\}=2 l_{n+m}+\frac{c}{3}\left(m^{2}-\frac{1}{4}\right) \delta_{m,-n},
$$


and

$$
\left[l_{m}, f_{n}\right]=\left(\frac{m}{2}-n\right) f_{m+n} .
$$

Again we impose that $c$ is central and as before we define $\mathscr{R}_{ \pm}=\mathscr{L}_{ \pm} \oplus \bigoplus_{ \pm n>0} \mathscr{F}_{n}$.

The ghost Fock space of the Ramond sector carries a representation of $\mathscr{R}$ with $c \mapsto-15 \mathrm{Id}, l_{n} \mapsto L_{n}^{\text {ghost }}, f_{n} \mapsto F_{n}^{\text {ghost }}$. The Fock space of the string oscillators also carries a representation of $\mathscr{R}$ with the opposite central charge (in the critical dimension) and where $l_{n} \mapsto L_{n}^{\text {matter }}$ and $f_{n} \mapsto F_{n}^{\text {matter }}$. Finally, let us denote by $L_{n}$ and $F_{n}$ the operators representing $l_{n}$ and $f_{n}$ respectively in the full Fock space (including ghosts). Again the formulas for these generators are standard and we refer the reader to [10].

In [11] Brower and Friedman claim to have proven full reducibility of this representation, although they do not write down the explicit spectrum generating algebra. Therefore, just as in the Neveu-Schwarz case we can decompose the string Fock space into Verma modules and thus restrict our attention to one such Verma module at a time when computing the BRST cohomology ${ }^{6}$

Let $\mathscr{M}$ be one such Verma module and let $H_{\infty}(\mathscr{R} ; \mathscr{M})$ denote the BRST cohomology $^{7}$ on the graded complex $C_{\infty}(\mathscr{R} ; \mathscr{M})=\bigoplus_{n} C_{\infty}^{n}(\mathscr{R} ; \mathscr{M})$, where again

$$
C_{\infty}^{n}(\mathscr{R} ; \mathscr{M})=C_{\infty}^{n}(\mathscr{R}) \otimes \mathscr{M}
$$

where $C_{\infty}^{n}(\mathscr{R})$ is the subspace of the ghost Fock space at ghost number $n$.

There are two natural subcomplexes to consider. One could consider the subcomplex relative to the zeroth subalgebra $\mathscr{R}_{0}=\mathscr{L}_{0} \oplus \mathscr{F}_{0}$ or relative to just the even part $\mathscr{L}_{0}$. The choice of subcomplex has to do the choice of Hilbert space $\mathscr{K}$ for the zero modes of the superconformal ghosts. The reason is the following. In order to consider the subcomplex relative to the full zeroth subalgebra we have to be able to impose the condition $\beta_{0} \omega=0$. This may or may not be possible as we shall now see.

The algebra obeyed by the ghost zero modes is the Heisenberg algebra

$$
\left[\gamma_{0}, \beta_{0}\right]=1
$$

and the hermiticity conditions are such that $\gamma_{0}$ is anti-hermitian and $\beta_{0}$ is hermitian. The unique ${ }^{8}$ representation of this algebra as operators in a Hilbert space (i.e. with a positive definite inner product) is the Schrödinger representation in which $\mathscr{K}$ is isomorphic with $\mathscr{L}^{2}(\mathbb{R}, d x)$ and where $\beta_{0}$ is represented by the multiplication operator: $\left(\beta_{0} h\right)(x)=x h(x)$ and $\gamma_{0}$ is $i$ times the momentum operator: $\left(\gamma_{0} h\right)(x)=h^{\prime}(x)$. If this is the case we cannot impose the equation $\beta_{0} \omega=0$ because the multiplication operator has no eigenvalues in $\mathscr{L}^{2}(\mathbb{R}, d x)$. In this case we would look at the subcomplex relative to $\mathscr{L}_{0}$.

\footnotetext{
${ }^{6}$ Just as before the vanishing theorem as it stands does not apply to the case where the center of mass momentum is zero. In this case the cohomology is again easy to compute explicitly

${ }^{7}$ Again this should be relative to the center. Therefore from now on $\mathscr{R}$ denotes the unextended Ramond algebra

${ }^{8}$ Strictly speaking the uniqueness is proven for the Weyl form of the Heisenberg algebra
} 
If on the other hand - like many other authors, notably Henneaux [12]-we treat $\gamma_{0}$ and $\beta_{0}$ as creation and annihilation operators (respectively) the Hilbert space is now (the completion of) the polynomial algebra in one variable $\mathbb{C}\left[\gamma_{0}\right]$. In this case the hermiticity conditions that induce a positive definite inner product are such that $\gamma_{0}$ and $\beta_{0}$ are mutually adjoint. In this case we can consider the subcomplex relative to the full zeroth subalgebra $\mathscr{R}_{0}$. It may seem unnatural to alter the hermiticity properties inherited from the classical fields, but for operators which do not correspond to physical observables the hermiticity properties are not too crucial. There is however a major drawback. Changing the hermiticity properties of $\gamma_{0}$ and $\beta_{0}$ changes the hermiticity properties of the BRST operator: it is no longer hermitian. This means that it is no longer guaranteed that the cohomology space inherits a well-defined (i.e., independent of the representative) inner product.

On the other hand keeping the original hermiticity conditions has one major inconvenience: the cohomology is not graded by ghost number and hence the vanishing theorem makes no sense. This is due essentially to the fact that the ghost number operator has no eigenvalues in $\mathscr{L}^{2}(\mathbb{R}, d x)$. Still, we can find a particular class of representatives which does admit a grading. In this case the cohomology agrees with the one obtained by altering the hermiticity properties of $\gamma_{0}$ and $\beta_{0}$, for which we can prove a vanishing theorem.

Therefore we will consider both choices of hermiticity properties. We will see that both cohomologies are isomorphic as ungraded vector spaces; and we will prove a vanishing theorem for the graded case.

\section{The Henneaux Representation}

Let us first assume that $\mathscr{K}=\mathbb{C}\left[\gamma_{0}\right]$. It is then possible to consider the relative subcomplex $C_{\infty}\left(\mathscr{R}, \mathscr{R}_{0} ; \mathscr{M}\right)$. This complex, which we abbreviate by $C_{\infty}$, is given by

$$
C_{\infty}=\left\{\omega \in C_{\infty}(\mathscr{R} ; \mathscr{M}) \mid F_{0} \omega=b_{0} \omega=\beta_{0} \omega=0\right\} .
$$

Just as in the Neveu-Schwarz case, it is finite dimensional. Hence a typical vector in $C_{\infty}$ is a linear combination of monomials

$$
|i, j, k, l, m, q\rangle=\prod_{n>0} \gamma_{-n}^{i_{n}} \prod_{n>0} \beta_{-n}^{j_{n}} \prod_{n>0} c_{-n}^{k_{n}} \prod_{n>0} b_{-n}^{l_{n}}|0\rangle \otimes \prod_{n>0} F_{-n}^{q_{n}} \prod_{n>0} L_{-n}^{m_{n}}|p\rangle,
$$

where $|p\rangle$ a highest weight vector of momentum $p$ such that

$$
\frac{1}{2} p^{2}=-\sum_{n}\left(i_{n}+j_{n}+q_{n}+k_{n}+l_{n}+m_{n}\right) n=-N,
$$

for some non-negative integer $N$. Define the filtration degree as

$$
\text { fdeg }|i, j, k, l, m, q\rangle=\sum_{n}\left(i_{n}-j_{n}-q_{n}+k_{n}-l_{n}-m_{n}\right) n .
$$

Just as in the Neveu-Schwarz case the filtration defined by this degree is bounded and defines a filtered complex. Therefore the theorem in Sect. 2 applies, yielding the existence of a spectral sequence which converges finitely to $H_{\infty}\left(\mathscr{R}, \mathscr{R}_{0} ; \mathscr{M}\right)$; and whose $E_{1}$ term is the differential for the complex

$$
C^{F_{0}}=\left(C\left(\mathscr{R}_{+}\right) \otimes C_{\infty}\left(\mathscr{R}_{-} ; \mathscr{M}\right)\right)^{F_{0}} .
$$


In this case, however, we cannot use the arguments used for the Neveu-Schwarz case because $F_{0}$ does not act reducibly. In fact, in the subspace left invariant by $L_{0}$, $F_{0}$ is nilpotent and not identically zero. Therefore $\operatorname{ker} F_{0} \cap \operatorname{im} F_{0} \neq 0$ and a decomposition à la (3.12) is impossible. Therefore we follow a completely different line of approach. We find a spectral sequence converging to $H\left(C^{F_{0}}\right)$ which preserves the grading by ghost number and for whose $E_{1}$ term we can prove a vanishing theorem.

The spectral sequence in question will be that associated to one of the canonical filtrations of a double complex. The double complex is constructed as follows. For any ghost number $p$ the space $\left(C^{p}\right)^{L_{0}}$ naturally affords a representation of $F_{0}$. Moreover since $F_{0}^{2}=L_{0}$ the action of $F_{0}$ is nilpotent and its cohomology may be defined. We define

$$
\mathbf{K}^{p, q}=C^{q}\left(\mathscr{F}_{0} ;\left(C^{p}\right)^{L_{0}}\right),
$$

the $q$-cochains of the $\mathscr{F}_{0}$ with coefficients in $\left(C^{p}\right)^{L_{0}}$. Let $\delta: \mathbf{K}^{p, q} \rightarrow \mathbf{K}^{p, q+1}$ to be the coboundary operator for $\mathscr{F}_{0}$ cochains. It is defined by

$$
\delta\left(f_{0}^{\prime}\right)^{q} \otimes \omega=\left(f_{0}^{\prime}\right)^{q+1} \otimes F_{0} \omega,
$$

for $\omega \in\left(C^{p}\right)^{L_{0}}$. Similarly define $d: \mathbf{K}^{p, q} \rightarrow \mathbf{K}^{p+1, q}$ to be the trivial extension of the differential $Q$ for $C^{L_{0}}$ :

$$
d\left(f_{0}^{\prime}\right)^{q} \otimes \omega=\left(f_{0}^{\prime}\right)^{q} \otimes Q \omega,
$$

for $\omega \in\left(C^{p}\right)^{L_{0}}$. Therefore the double complex can be represented as follows:

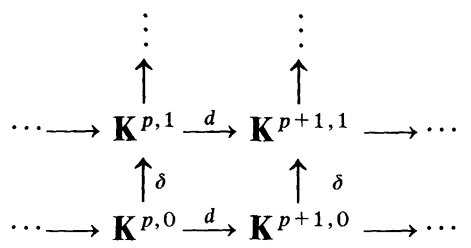

Since $Q$ and $F_{0}$ anticommute so do $d$ and $\delta$. Therefore $D=d+\delta$ is nilpotent and computes the cohomology of the total complex $\mathbf{K}=\bigoplus_{m} \mathbf{K}^{m}$ where $\mathbf{K}^{m}=\bigoplus_{p} \mathbf{K}^{p, m-p}$.

Because $C^{L_{0}}$ is finite-dimensional its grading by ghost number is bounded and therefore the total complex is finite in each dimension. Therefore we can use the results of Sect. 2 and deduce that there exist two spectral sequences converging to the total cohomology in each dimension. We now compute the early terms. We first look at the vertical $\delta$ cohomology. The space $Z_{\delta}^{p, q}$ of $(p, q)$-cocycles of $\delta$ is just $\left(f_{0}^{\prime}\right)^{q} \otimes\left(C^{p}\right)^{L_{0}}$ whereas the $(p, q)$-coboundaries are $\left(f_{0}^{\prime}\right)^{q} \otimes F_{0}\left(C^{p}\right)^{L_{0}}$ for $q>0$ whereas for $q=0$ there are no coboundaries since there are no -1 cochains. Therefore the vertical cohomology is

$$
H_{\delta}^{p, q}= \begin{cases}1 \otimes\left(C^{p}\right)^{F_{0}} & \text { for } q=0 \\ \left(f_{0}^{\prime}\right)^{q} \otimes H_{F_{0}}\left(\left(C^{p}\right)^{L_{0}}\right) & \text { for } q \neq 0,\end{cases}
$$

where $H_{F_{0}}\left(\left(C^{p}\right)^{L_{0}}\right)$ is the cohomology of the nilpotent operator $F_{0}$ in $\left(C^{p}\right)^{L_{0}}$. This 
space, however, turns out to be trivial [12]. In fact, one can ${ }^{9}$ define an operator $K$ such that $\left\{F_{0}, K\right\}=1$. Therefore the vertical cohomology is zero except in dimension zero where it is isomorphic to $C^{F_{0}}$.

The spectral sequence associated to the horizontal filtration has as ' $E_{1}$ term the vertical cohomology and as ' $E_{2}$ term $H_{d}\left(H_{\delta}\right)$. Therefore this is zero everywhere but in dimension zero and there it is just $H\left(C^{F_{0}}\right)$. Because $d_{2}$ maps already between different rows we see that it is identically zero and so are all the higher $d_{r}$ 's. Hence the spectral sequence collapses and we have that the total cohomology is

$$
H_{D}^{m} \cong H^{m}\left(C^{F_{0}}\right) \text {. }
$$

If we take the vertical filtration the first term in the spectral sequence is the horizontal cohomology $H_{d}$. Therefore the " $E_{1}$ is precisely

$$
" E_{1}^{q, p}=\left(f_{0}^{\prime}\right)^{q} \otimes H^{p}\left(C^{L_{0}}\right),
$$

where by an argument identical to that in the Neveu-Schwarz case we can show that $H^{p}\left(C^{L_{0}}\right) \cong H^{p}(C)^{L_{0}}$. By arguments identical to the ones in the Neveu-Schwarz sector - i.e., using the Künneth formula and the theorem in Appendix A - it follows that $H^{p}(C)^{L_{0}}$ is zero for $p<0$. Therefore

$$
" E_{1}^{m} \cong \bigoplus_{q \geqq 0} H^{m-q}\left(C^{L_{0}}\right)
$$

Since $H^{p}\left(C^{L_{0}}\right)=0$ for $p<0$ we have that " $E_{1}^{m}=0$ for $m<0$. Therefore " $E_{\infty}^{m}=0$ for $m<0$. But by the theorem in Sect. 2, this limit term is also the total cohomology. Therefore $H^{m}\left(C^{F_{0}}\right)=0$ for $m<0$. But this is the $E_{1}$ term in a spectral sequence converging to $H_{\infty}\left(\mathscr{R}, \mathscr{R}_{0} ; \mathscr{M}\right)$. Therefore we conclude that $H_{\infty}^{m}\left(\mathscr{R}, \mathscr{R}_{0} ; \mathscr{M}\right)=0$ for $m<0$ and the same for $H_{\infty}\left(\mathscr{R}, \mathscr{R}_{0} ; \mathscr{H}\right)$. By the Poincare duality of [4], this implies the vanishing theorem

$$
H_{\infty}^{m \neq 0}\left(\mathscr{R}, \mathscr{R}_{0} ; \mathscr{H}\right)=0
$$

We will see in the next section that this implies a vanishing theorem for the cohomology of the full complex $C_{\infty}(\mathscr{R} ; \mathscr{H})$

\section{The Schrödinger Representation}

Now let us assume that $\mathscr{K}=\mathscr{L}^{2}(\mathbb{R}, d x)$. We find it convenient to work in a dense domain in which $\gamma_{0}$ and $\beta_{0}$ are defined. To this end let us introduce the operators $a$ and $a^{\dagger}$ defined by

$$
\beta_{0}=\frac{1}{\sqrt{2}}\left(a^{\dagger}-a\right), \quad \gamma_{0}=\frac{1}{\sqrt{2}}\left(a^{\dagger}+a\right),
$$

and let $\mathscr{K}$ be the completion of the polynomial algebra $\mathbb{C}\left[a^{\dagger}\right]$. Combining (4.4) and (4.18) we find that $a$ and $a^{\dagger}$ obey $\left[a, a^{\dagger}\right]=1$.

Let us define the subcomplex

$$
C_{\infty}\left(\mathscr{R}, \mathscr{L}_{0} ; \mathscr{M}\right)=\left\{\omega \in C_{\infty}(\mathscr{R} ; \mathscr{M}) \mid L_{0} \omega=b_{0} \omega=0\right\} .
$$

\footnotetext{
${ }^{9}$ Strictly speaking, this in only possible for states whose center of mass momentum is different from zero. In the Ramond sector any such on-shell ( $L_{0}$ invariant) states correspond to one of the degenerate vacua and hence it has manifestly zero ghost number
} 
To study the cohomology of this complex it is convenient to discuss the differentials occurring in the various complexes under study. The differential in the full complex $C_{\infty}(\mathscr{R} ; \mathscr{M})$ is the BRST operator $Q$. Making the dependence on the ghosts' zero modes manifest we can write it as

where

$$
Q=c_{0} L_{0}-2 b_{0} T-\gamma_{0}^{2} b_{0}+\mathbb{Q},
$$

$$
\mathbb{Q}=\beta_{0} K+\gamma_{0} F_{0}+\mathbb{Q} \text {. }
$$

We don't need the explicit expressions for these operators but only the following relations which follow from the nilpotency of $Q$ :

$$
\mathbb{Q}^{2}=0 \quad F_{0}^{2}=L_{0} \quad\left[F_{0}, T\right]=K \quad Q^{2}=2 L_{0} T+F_{0} K,
$$

and all other (anti)commutators vanish; in particular, $[T, K]=0$.

The differential in the relative subcomplex $C_{\infty}\left(\mathscr{R}, \mathscr{L}_{0} ; \mathscr{M}\right)$ is $\mathbb{Q}$. Isolating the representation space of the superghosts' zero modes, this subcomplex can be written as $C \otimes \mathbb{C}\left[a^{\dagger}\right]$ which defines $C$. According to this decomposition the differential becomes

$$
\mathbb{Q}=\mathbb{Q} \otimes \mathrm{Id}+\frac{1}{\sqrt{2}}\left(F_{0}+K\right) \otimes a^{\dagger}+\frac{1}{\sqrt{2}}\left(F_{0}-K\right) \otimes a
$$

In this subcomplex the following identities are satisfied

$$
F_{0}^{2}=0 \quad Q^{2}=F_{0} K .
$$

Hence the space $C^{F_{0}}$ is a differential complex with respect to $\mathscr{Q}$. Notice that this complex is isomorphic to $C_{\infty}\left(\mathscr{R}, \mathscr{R}_{0} ; \mathscr{M}\right)$ in the Henneaux representation. Therefore their cohomologies are isomorphic as well. We will now prove that the cohomology of this complex, denoted by $H_{\mathscr{Q}}\left(C^{F_{0}}\right)$ is isomorphic to $H_{\infty}\left(\mathscr{R}, \mathscr{L}_{0} ; \mathscr{M}\right)$. But first we need a preliminary result.

Because $\left[F_{0}, T\right]=K$ and $[T, K]=0$ we can write

$$
F_{0}+K=e^{-T} F_{0} e^{T},
$$

which is well defined as it stands because $C$ is finite dimensional. Also because $C$ is finite dimensional any operator with non-zero ghost number ${ }^{10}$ is automatically nilpotent. In particular, since $T$ has ghost number 2 , it is nilpotent and therefore $\exp (\alpha T)$ is an isomorphism for any complex number $\alpha$. Because $F_{0}$ is nilpotent, $F_{0}+K$ is also nilpotent and its cohomology is isomorphic to that of $F_{0}: \exp (-T)$ gives the isomorphism by (4.25). Since the cohomology of $F_{0}$ is trivial, as proven in [12], so is the cohomology of $F_{0}+K$.

We now proceed to prove the isomorphism of $H_{\mathscr{Q}}\left(C^{F_{0}}\right)$ and $H_{\infty}\left(\mathscr{R}, \mathscr{L}_{0} ; \mathscr{M}\right)$. Let $\Psi$ be a cocycle in $C \otimes \mathbb{C}\left[a^{\dagger}\right]$. Then we can write it as a polynomial with coefficients in $C$ as follows

$$
\Psi=\sum_{n=0}^{N} \psi_{n} \otimes\left(a^{\dagger}\right)^{n}
$$

where $\psi_{n} \in C$ for all $n$. Then the fact that it is a cocycle implies that $\left(F_{0}+K\right) \psi_{N}=0$. By the vanishing of the cohomology of $F_{0}+K$ there exists a cochain $\phi$ such that $\psi_{N}+\left(F_{0}\right.$

\footnotetext{
${ }^{10}$ Here ghost number does not take into account the zero modes
} 
$+K) \phi=0$. Therefore adding the coboundary $\mathbb{Q}\left(\phi \otimes\left(a^{\dagger}\right)^{N-1}\right)$ to $\Psi$ we get rid of the $N^{\text {th }}$ order term in $\Psi$. Continuing in this fashion we can reduce $\Psi$ to a constant monomial $\psi \otimes 1$, which is still a cocycle cohomologous to $\Psi$. The fact that it is a cocycle implies that $Q \psi Q=0$ and $\left(F_{0}+K\right) \psi=0$. Therefore, using the fact that $[T, Q]$ $=0$, we see that $\exp (T) \psi$ obeys

$$
\mathbb{Q} e^{T} \psi=0 \quad F_{0} e^{T} \psi=0,
$$

hence it defines a class $[\exp (T) \psi]$ in $H_{Q}\left(C^{F_{0}}\right)$. It is straightforward to verify that if this class is trivial then the class $[\Psi]$ in $H_{\infty}\left(\mathscr{R}, \mathscr{L}_{0} ; \mathscr{M}\right)$ is also trivial. Therefore we have an injection $H_{\infty}\left(\mathscr{R}, \mathscr{L}_{0} ; \mathscr{M}\right) \hookrightarrow H_{\mathscr{Q}}\left(C^{F_{0}}\right)$.

We now prove the reverse injection. Let $\psi$ define a class in $H_{\mathscr{Q}}\left(C^{F_{0}}\right)$. Then $[\exp (-T) \psi \otimes 1]$ defines a class in $H_{\infty}\left(\mathscr{R}, \mathscr{L}_{0} ; \mathscr{M}\right)$. Now suppose that this class is trivial; that is,

$$
e^{-T} \psi \otimes 1=\mathbb{Q} \Xi \text { for some } \Xi
$$

Just as before we may add coboundaries to $\Xi$ in such a way that (4.28) is still obeyed and such that $\Xi$ gets reduced to a constant monomial $\xi \otimes 1$. In that case, $F_{0} \exp (T) \xi$ $=0$ and $\psi=Q \exp (T) \xi$; whence $[\psi]=0$. This gives the reverse injection and concludes the proof of the isomorphism.

Notice that the isomorphism is only an isomorphism of ungraded vector spaces. In particular the cohomology space $H_{\infty}\left(\mathscr{R}, \mathscr{L}_{0} ; \mathscr{M}\right)$ is not graded by ghost number since the ghost number operator on $\mathbb{C}\left[a^{\dagger}\right]$ is of the form $\frac{1}{2}\left(\left(a^{\dagger}\right)^{2}-a^{2}\right)$ and therefore has no eigenvalues. As a consequence, a vanishing theorem has no meaning in this representation. This is not a serious drawback when it comes to proving the "noghost" theorem as we shall see, although it takes away some of the structure.

One can also show that every cohomology class in $\mathscr{H}_{\infty}\left(\mathscr{R}, \mathscr{L}_{0} ; \mathscr{M}\right)$ has at least one representative of ghost number zero. This uses a straightforward generalization for the NSR string of a result proven in [13] for the open bosonic string which states that every cohomology class in $H_{Q}\left(C^{F_{0}}\right)$ has a representative annihilated by $T$. If this is the case then it is also annihilated by $K$ and therefore it defines a class in $H_{\infty}\left(\mathscr{R}, \mathscr{L}_{0} ; \mathscr{M}\right)$; and by the vanishing theorem for $H_{\mathscr{Q}}\left(C^{F_{0}}\right)$ it has ghost number zero.

\section{Vanishing Theorems for the Full Complexes}

In this section we prove vanishing theorems for the cohomology of the full complexes $C_{\infty}(\mathscr{R} ; \mathscr{H})$ and $C_{\infty}(\mathscr{N} \mathscr{S} ; \mathscr{H})$. For the Ramond sector we only work with the Henneaux representation since for the Schrödinger representation there is no vanishing theorem. First we will prove that

$$
H_{\infty}\left(\mathscr{R}, \mathscr{L}_{0} ; \mathscr{H}\right) \cong H_{\infty}\left(\mathscr{R}, \mathscr{R}_{0} ; \mathscr{H}\right)
$$

Then we will prove that

$$
H_{\infty}^{n}(\mathscr{N} \mathscr{S} ; \mathscr{H}) \cong \begin{cases}H_{\infty}^{0}\left(\mathscr{N} \mathscr{S}, \mathscr{L}_{0} ; \mathscr{H}\right) & \text { for } n= \pm \frac{1}{2} \\ 0 & \text { otherwise }\end{cases}
$$


and

$$
H_{\infty}^{n}(\mathscr{R} ; \mathscr{H}) \cong \begin{cases}H_{\infty}^{0}\left(\mathscr{R}, \mathscr{L}_{0} ; \mathscr{H}\right) & \text { for } n= \pm \frac{1}{2} \\ 0 & \text { otherwise }\end{cases}
$$

Several remarks are in order before we start proving these results. The first is to notice the rather surprising fact that the BRST cohomology of the Ramond sector has the same finite degeneracy as the one of the Neveu-Schwarz sector despite the fact that at the level of cochains the Ramond sector is infinitely degenerate due to the existence of the zero modes for the superconformal ghosts. Secondly we notice that the grading of the full complex is half integral. This is the choice that makes the full ghost number operator hermitian. Thirdly, because the proofs of (5.2) and (5.3) are virtually identical we will only present the one for the Ramond sector: this being the more involved of the two. Finally, the proof of (5.1) is similar to the proof of the isomorphisms of the relative BRST cohomology of the Ramond sector in the Henneaux and Schrödinger representations. In fact, part of the proof already appears in [12].

With these remarks behind us we proceed with the proofs.

Proof of (5.1). Let us isolate the space in which the zero modes of the superconformal ghosts act by writing $C_{\infty}\left(\mathscr{R}, \mathscr{L}_{0} ; \mathscr{H}\right)$ as $C^{\mathscr{L}_{0}} \otimes \mathbb{C}\left[\gamma_{0}\right]$, which defines $C$. Then $C_{\infty}\left(\mathscr{R}, \mathscr{R}_{0} ; \mathscr{H}\right)$ may be identified with $C^{F_{0}}$ and embedded in $C_{\infty}\left(\mathscr{R}, \mathscr{L}_{0} ; \mathscr{H}\right)$ as $C^{F_{0}} \otimes 1$. That is, if $\psi \in C_{\infty}\left(\mathscr{R}, \mathscr{R}_{0} ; \mathscr{H}\right)$, then $\psi \otimes 1 \in C_{\infty}\left(\mathscr{R}, \mathscr{L}_{0} ; \mathscr{H}\right)$. Suppose that $\Psi$ is a cocycle in $C_{\infty}\left(\mathscr{R}, \mathscr{L}_{0} ; \mathscr{H}\right)$. Then $\Psi$ is a polynomial in $\gamma_{0}$ with coefficients in $C^{L_{0}}$

$$
\Psi=\sum_{n=0}^{N} \psi_{n} \otimes \gamma_{0}^{n} \quad \psi_{n} \in C^{L_{0}} \forall n
$$

such that, in particular, $F_{0} \psi_{N}=0$. Since the cohomology of $F_{0}$ is trivial, there exists $\phi \in C^{L_{0}}$ such that $\psi_{N}+F_{0} \phi=0$. Therefore $\Psi+\mathbb{Q}\left(\phi \otimes \gamma_{0}^{N-1}\right)$ is a cocycle cohomologous to $\Psi$ but lacking the highest order term in $\gamma_{0}$. Continuing in this fashion we can reduce $\Psi$ to a constant monomial $\psi \otimes 1$ still cohomologous to $\Psi$. The cocycle condition translates into

$$
Q \mathcal{Q} \psi=0 \quad F_{0} \psi=0 ;
$$

hence it defines a class in $H_{\infty}\left(\mathscr{R}, \mathscr{R}_{0} ; \mathscr{H}\right)$. Suppose that this class is trivial; that is, $\psi=Q \mathcal{C} \zeta$ where $F_{0} \zeta=0$. Then $\psi \otimes 1=\mathbb{Q}(\zeta \otimes 1)$ and thus $\Psi$ represents the trivial class. Therefore we have an injection $H_{\infty}\left(\mathscr{R}, \mathscr{L}_{0} ; \mathscr{H}\right) \hookrightarrow H_{\infty}\left(\mathscr{R}, \mathscr{R}_{0} ; \mathscr{H}\right)$.

Conversely, let $\psi$ be a cocycle in $H_{\infty}\left(\mathscr{R}, \mathscr{R}_{0} ; \mathscr{H}\right)$. Then $\psi \otimes 1$ defines a class in $H_{\infty}\left(\mathscr{R}, \mathscr{L}_{0} ; \mathscr{H}\right)$. If trivial,

$$
\psi \otimes 1=\mathbb{Q} \Xi
$$

for some polynomial $\Xi=\sum_{n=0}^{N} \xi_{n} \otimes \gamma_{0}^{n}$. In particular, (5.6) implies that $F_{0} \xi_{N}=0$. As before there exists $\lambda$ such that $\xi_{N}+F_{0} \lambda=0$. Thus $\Xi+\mathbb{Q}\left(\lambda \otimes \gamma_{0}^{N-1}\right)$ still obeys (5.6) but has no order $N$ term. Continuing in this way we can reduce $\Xi$ to a constant monomial $\xi \otimes 1$ still obeying (5.6). In particular, this implies that

$$
Q \xi=\psi \text { and } F_{0} \xi=0 \text {. }
$$


Therefore $\psi$ defines the trivial class in $H_{\infty}\left(\mathscr{R}, \mathscr{R}_{0} ; \mathscr{H}\right)$. This proves the reverse injection and hence the isomorphism (5.1).

In order to prove (5.3) and because $\mathscr{L}_{0}$ acts diagonally in the relative subcomplex $C_{\infty}\left(\mathscr{R}, \mathscr{L}_{0} ; \mathscr{H}\right)$ we could appeal to a suitably generalized result of Koszul [14] which asserts the existence of a spectral sequence converging to $H_{\infty}(\mathscr{R} ; \mathscr{H})$ whose $E_{2}$ term is

$$
H_{\infty}\left(\mathscr{R}, \mathscr{L}_{0} ; \mathscr{H}\right) \otimes H\left(\mathscr{L}_{0}\right)
$$

This, together with the easily verifiable fact that

$$
H^{n}\left(\mathscr{L}_{0}\right) \cong \begin{cases}\mathbb{C} & \text { for } n= \pm \frac{1}{2} \\ 0 & \text { otherwise }\end{cases}
$$

and the fact that - due to the vanishing theorem for $H_{\infty}\left(\mathscr{R}, \mathscr{L}_{0} ; \mathscr{H}\right)$ - the spectral sequence collapses at the $E_{2}$ term, yields (5.3).

However we can arrive at the same result in a slightly more pedestrian way by using the spectral sequence associated to a particular double complex.

Proof of (5.3). The differential in the complex $C_{\infty}(\mathscr{R} ; \mathscr{H})$ is the full BRST operator given by (4.20) where $\mathbb{Q}$, the differential in the complex $C_{\infty}\left(\mathscr{R}, \mathscr{L}_{0} ; \mathscr{H}\right)$, is given by (4.21). For notational convenience we define $\mathscr{T}=-2\left(T+\frac{1}{2} \gamma_{0}^{2}\right)$. Notice that since $L_{0}$ is diagonalizable and null homotopic: $L_{0}=\left\{Q, b_{0}\right\}$, we can restrict ourselves to $L_{0}$-invariants. Therefore we write the differential in $C_{\infty}(\mathscr{R} ; \mathscr{H})$ as

$$
Q=\mathbb{Q}+b_{0} \mathscr{T},
$$

where, due to the nilpotency of $Q, \mathbb{Q}$ and $b_{0}$, both terms anticommute. Abbreviating $C_{\infty}(\mathscr{R} ; \mathscr{H})^{L_{0}}$ to $C$, let us define a tri-grading on this complex as follows:

$$
C=\bigoplus_{m \in \mathbb{Z}} \bigoplus_{n= \pm \frac{1}{2}} \bigoplus_{p \in \mathbb{Z}+\frac{1}{2}} C^{m, n, p},
$$

where $C^{m, n, p}$ consists of those cochains which are tensor products of homogeneous terms of "reduced" ghost number $m,\left(b_{0}, c_{0}\right)$-ghost number $n$ and $\left(\beta_{0}, \gamma_{0}\right)$-ghost number $p$. By "reduced" ghost number we mean the ghost number which grades the relative subcomplex $C_{\infty}\left(\mathscr{R}, \mathscr{R}_{0} ; \mathscr{H}\right)$.

According to this tri-grading the relevant terms appearing in $Q$ have the following tri-degree:

\begin{tabular}{ll}
\hline Term & Tri-degree \\
\hline$Q$ & $(1,0,0)$ \\
$\beta_{0} K$ & $(2,0,-1)$ \\
$\gamma_{0} F_{0}$ & $(0,0,1)$ \\
$b_{0} T$ & $(2,-1,0)$ \\
$b_{0} \gamma_{0}^{2}$ & $(0,-1,2)$ \\
\hline
\end{tabular}

Defining the bigraded complex $K=\bigoplus_{r, s} K^{r, s}$ by

$$
K^{r, s}=\bigoplus_{m+p=r} C^{m, s, p}
$$


we notice that $\mathbb{Q}$ has bidegree $(1,0)$ but that $b_{0} \mathscr{T}$ has bidegree $(2,-1)$. Hence the complex as it stands is slightly skewed. Making a last redefinition, let us introduce another bigraded complex $\mathbf{K}$ which is just a relabeling of $K$ by $\mathbf{K}=\bigoplus_{p, q} \mathbf{K}^{p, q}$, where

$$
\mathbf{K}^{p, q}=K^{p+2 q,-q} .
$$

Then $\mathbb{Q}: \mathbf{K}^{p, q} \rightarrow \mathbf{K}^{p+1, q}$ and $b_{0} \mathscr{T}: \mathbf{K}^{p, q} \rightarrow \mathbf{K}^{p, q+1}$ yielding a double complex. Decomposing this double complex into eigenspaces of the level operator (the momentum independent part of $L_{0}$ ) we easily see that it yields an infinite direct sum of finite double complexes. Proving (5.3) for each subcomplex and then collating all terms proves (5.3) for the full complex. Hence from now on we are working in a given eigenspace of the level operator so that the double complex $\mathbf{K}$ is finite. Notice that the complex is only two rows high in any case, since $q$ only takes $\pm \frac{1}{2}$ as values.

As discussed in Sect. 2 we have two canonical spectral sequences associated to this double complex. We use the "filtration. Its $E_{1}$ term is the horizontal cohomology for which we have a vanishing theorem. Keeping track of the gradings we have

$$
" E_{1}^{p, q} \cong \begin{cases}H_{\infty}^{0}\left(\mathscr{R}, \mathscr{L}_{0} ; \mathscr{H}\right) & \text { for }(p, q)=\left(-1, \frac{1}{2}\right) \text { and }\left(1,-\frac{1}{2}\right) \\ 0 & \text { otherwise }\end{cases}
$$

Notice further that $d_{1}$ is identically zero since it maps vertically and by (5.14) its domain or its range is zero in all cases. Furthermore all higher $d_{r}$ are also zero because they skip at least one row and there are only two rows in the complex. Therefore the $E_{1}$ term is the limit term which is the cohomology of the full complex: $H_{\infty}(\mathscr{R} ; \mathscr{H})$. This proves $(5.3)$.

As remarked earlier the proof of (5.2) follows the same steps as the proof of (5.3), but without the complications arising from the superconformal ghosts.

\section{6. "No-Ghost" Theorems}

In this section we prove the "no-ghost" theorem for the NSR string along the lines suggested in [4]. This method was used to prove the similar result for the bosonic string in [1] and [3]. We briefly recall the method.

Let $C_{\infty}$ denote the appropriate relative subcomplex of the string. We use the relative subcomplexes since the full complex - as we have seen in the previous section - is just two copies of the relative one; hence proving positive-definiteness of the inner product in the relative subcomplex suffices. Let $\mathscr{C}$ denote the conjugation used to redefine the inner product in order to make it positive definite. The existence of this positive definite inner product allowed us to define a BRST laplacian whose kernel $\mathbf{H}$ is isomorphic to the BRST cohomology. Because $\mathscr{C}$ commutes with the laplacian it stabilizes its kernel. Moreover since $\mathscr{C}$ reverses ghost number it stabilizes also $\mathbf{H}_{0}$ which is isomorphic to the physical space defined as the zeroth BRST cohomology space. From its definition (see [4] for the details and Appendix B for its explicit construction in this case) $\mathscr{C}$ is the identity on states of positive norm and 
minus the identity on states of negative norm. Therefore we see that ${ }^{11}$

$$
\operatorname{Tr}_{\mathbf{H}_{0}} \mathscr{C} \leqq \operatorname{dim} \mathbf{H}_{0}
$$

where the bound is saturated if and only if $\mathbf{H}_{0}$ is positive definite. Since the inner product on the cohomology does not depend on the particular representative, the saturation of the above bound is equivalent to the "no-ghost" theorem.

As they stand, the quantities in (6.1) are hard to calculate. However, since $\mathscr{C}$ $\operatorname{maps} \mathbf{H}_{p} \rightarrow \mathbf{H}_{-p}$,

$$
\operatorname{Tr}_{\mathbf{H}_{0}} \mathscr{C}=\operatorname{Tr}_{\mathbf{H}} \mathscr{C}
$$

Moreover in the Hodge style decomposition [4] of $C_{\infty}$ as $\mathbf{H} \oplus \mathrm{im} Q \oplus \mathrm{im} Q^{*}$, where $Q^{*}$ is the adjoint of $Q$ with respect to the positive definite inner product, $\mathscr{C}$ maps $\operatorname{im} Q \rightarrow \operatorname{im} Q^{*}$ and back. Since the trace is basis independent we see that

$$
\operatorname{Tr}_{\mathbf{H}_{0}} \mathscr{C}=\operatorname{Tr}_{C_{\infty}} \mathscr{C}
$$

which is easy enough to calculate. This settles the left-hand side of (6.1). As for the right-hand side we notice, using the vanishing theorem, that $\operatorname{dim} \mathbf{H}_{0}$ is nothing but the Euler characteristic $\chi\left(C_{\infty}\right)$ of the relative subcomplex : the alternating sum of the dimensions of the cohomology spaces. Using the Euler-Poincaré principle we can write the Euler characteristic as $\operatorname{Tr}_{C_{\infty}}(-1)^{\mathscr{G}}$ where $\mathscr{G}$ is the ghost number operator in the relative subcomplex. This again is quite straightforward to compute. Therefore (6.1) is equivalent to

$$
\operatorname{Tr}_{C_{\infty}} \mathscr{C} \leqq \operatorname{Tr}_{C_{\infty}}(-1)^{\mathscr{G}}
$$

Our proof of the "no-ghost" theorem will consist in proving that the above bound is saturated for the NSR string. Since the relative subcomplex is graded by the level operator $\mathscr{L}$ (the momentum independence piece of $L_{0}$ ) and each level eigenspace is finite dimensional the following converges for $q$ sufficiently small

$$
\operatorname{Tr}_{C_{\infty}} q^{\mathscr{L}} \mathscr{C} \leqq \operatorname{Tr}_{C_{\infty}} q^{\mathscr{L}}(-1)^{\mathscr{G}}
$$

Because $C_{\infty}$ splits as tensor products corresponding to the different oscillators and the trace is multiplicative over the tensor product, we compute each term separately and then multiply the results. There are two terms common to both sectors: the $\{\alpha\}$ and $\{b, c\}$ oscillators; and we do these now. This calculation was done in [1] and [3] (for $D=26$ ) but we repeat it here (for $D=10$ ) for completeness.

The space over which we are taking the traces has the following structure

$$
C=\bigotimes_{\mu=0}^{9} \bigotimes_{n=1}^{\infty} S_{n}^{\mu} \bigotimes_{n=1}^{\infty} A_{n},
$$

where $S_{n}^{\mu}$ is the one particle Hilbert space corresponding to the oscillator $a_{n}^{\mu \dagger}$ and $A_{n}$ is the Hilbert space corresponding to the oscillators $\left\{b_{n}^{\dagger}, c_{n}^{\dagger}\right\}$. The space $S_{n}^{\mu}$ is isomorphic to the polynomial algebra in one variable: $a_{n}^{\mu \dagger}$ whereas the space $A_{n}$ is isomorphic to the exterior algebra on two generators: $b_{n}^{\dagger}$ and $c_{n}^{\dagger}$.

\footnotetext{
${ }^{11}$ As it stands this next equation is ill-defined since $\mathbf{H}_{0}$ is infinite dimensional. These quantities are to be understood as weighted traces; the dimension being understood as the trace of the identity
} 
Therefore using the fact that the trace is multiplicative over tensor products the right-hand side of (6.5) becomes

$$
\begin{aligned}
\operatorname{Tr}_{C}(-1)^{\mathscr{G}} q^{\mathscr{L}} & =\prod_{\mu=0}^{9} \prod_{n=1}^{\infty} \operatorname{Tr}_{S_{n}^{\mu}} q^{n a_{n}^{\mu \dagger} a_{n}^{\mu}} \times \prod_{n=1}^{\infty} \operatorname{Tr}_{A_{n}}\left[(-1)^{c_{n}^{\dagger} b_{n}-b_{n}^{\dagger} c_{n}} q^{n\left(c_{n}^{\dagger} b_{n}+b_{n}^{\dagger} c_{n}\right)}\right] \\
& =\left[\prod_{n=1}^{\infty}\left(\sum_{m=0}^{\infty} q^{n m}\right)\right]^{10} \times \prod_{n=1}^{\infty}\left(1-q^{n}-q^{n}+q^{2 n}\right) \\
& =\prod_{n=1}^{\infty}\left(1-q^{n}\right)^{-10} \cdot\left(1-q^{n}\right)^{2}=\prod_{n=1}^{\infty}\left(1-q^{n}\right)^{-8}
\end{aligned}
$$

As for the left-hand side we have

$$
\begin{aligned}
\operatorname{Tr}_{C} \mathscr{C} q^{\mathscr{L}} & =\prod_{\mu=0}^{9} \prod_{n=1}^{\infty} \operatorname{Tr}_{S_{n}^{\mu}} \mathscr{C} q^{n a_{n}^{\mu \dagger} a_{n}^{\mu}} \times \prod_{n=1}^{\infty} \operatorname{Tr}_{A_{n}} \mathscr{C} q^{n\left(c_{n}^{\dagger} b_{n}+b_{n}^{\dagger} c_{n}\right)} \\
& =\prod_{\mu=0}^{9} \prod_{n=1}^{\infty} \sum_{m=0}^{\infty}\left((-1)^{\delta_{\mu, 0}} q^{n}\right)^{m} \times \prod_{n=1}^{\infty}\left(1-q^{2 n}\right) \\
& =\prod_{n=1}^{\infty}\left(1+q^{n}\right)^{-1} \cdot\left(1-q^{n}\right)^{-9} \cdot\left(1-q^{n}\right) \cdot\left(1+q^{n}\right)=\prod_{n=1}^{\infty}\left(1-q^{n}\right)^{-8} .
\end{aligned}
$$

We see already that the identity is satisfied. This is not surprising since this is essentially the "no-ghost" theorem for the bosonic string. Of course, in this case, the calculation has no cohomological significance since we are away from the critical dimension.

Having done the calculations common to both sectors we now do each sector separately.

\section{The Neveu-Schwarz Sector}

The relative subcomplex $C_{\infty}\left(\mathscr{N} \mathscr{S}, \mathscr{L}_{0} ; \mathscr{H}\right)$, which we abbreviate to $C_{\infty}$, has the following structure

where

$$
C_{\infty}=\mathscr{H}^{(a)} \otimes \mathscr{H}^{(b, c)} \otimes \mathscr{H}^{(b)} \otimes \mathscr{H}^{(\beta, \gamma)}
$$

$$
\begin{gathered}
\mathscr{H}^{(a)}=\bigotimes_{\mu=0}^{9} \bigotimes_{n=1}^{\infty} S_{n}^{\mu}, \\
\mathscr{H}^{(b, c)}=\bigotimes_{n=1}^{\infty} A_{n}, \\
\mathscr{H}^{(b)}=\bigotimes_{\mu=0}^{9} \bigotimes_{r=\frac{1}{2}}^{\infty} A_{r}^{\mu},
\end{gathered}
$$

and

$$
\mathscr{H}^{(\beta, \gamma)}=\bigotimes_{r=\frac{1}{2}}^{\infty} S_{r}
$$

The first two terms are the ones over which we computed the relevant traces in the beginning of this section. Therefore we shall concentrate on the last two terms. Here $A_{r}^{\mu}$ is the Hilbert space of the $b_{r}^{\mu \dagger}$ oscillator and is isomorphic to the exterior algebra 
on one generator; and $S_{r}$ is the Hilbert space of the $\left\{\beta_{r}^{\dagger}, \gamma_{r}^{\dagger}\right\}$ oscillators and is isomorphic to the polynomial algebra in two variables.

The contribution to the right-hand side of (6.5) coming from the first two terms in the above decomposition are $\prod_{n=1}^{\infty}\left(1-q^{n}\right)^{-8}$. The contribution coming from the Neveu-Schwarz oscillators can be computed as follows

$$
\operatorname{Tr}_{\mathscr{H}(b)} q^{\mathscr{L}^{(b)}}=\prod_{\mu=0}^{9} \prod_{r=\frac{1}{2}}^{\infty} \operatorname{Tr}_{A_{r}^{\mu}} q^{r b_{r}^{\mu \dagger} b_{r}^{\mu}}=\prod_{r=\frac{1}{2}}^{\infty}\left(1+q^{r}\right)^{10}
$$

whereas the contribution from the superghosts is

$$
\begin{aligned}
\operatorname{Tr}_{\mathscr{H}^{(\beta, \gamma)}(-1)^{\mathscr{G}} q^{\mathscr{L}^{(\beta, \gamma)}}} & =\prod_{r=\frac{1}{2}}^{\infty} \operatorname{Tr}_{S_{r}}(-1)^{N_{\gamma}-N_{\beta}} q^{r\left(N_{\gamma}+N_{\beta}\right)} \\
& =\prod_{r=\frac{1}{2}}^{\infty} \operatorname{Tr}_{S_{r}}\left(-q^{r}\right)^{N_{\gamma}+N_{\beta}}=\prod_{r=\frac{1}{2}}^{\infty} \sum_{n, m=0}^{\infty}\left(-q^{r}\right)^{n+m} \\
& =\prod_{r=\frac{1}{2}}^{\infty}\left(\sum_{n=0}^{\infty}\left(-q^{r}\right)^{n}\right)^{2}=\prod_{r=\frac{1}{2}}^{\infty}\left(1+q^{r}\right)^{-2},
\end{aligned}
$$

where $N_{\beta}$ (respectively $N_{\gamma}$ ) is the number operator corresponding to the $\left\{\beta_{r}\right\}$ (respectively $\left\{\gamma_{r}\right\}$ ) oscillators. Putting erverything together we find that

$$
\operatorname{Tr}_{C_{\infty}}(-1)^{\mathscr{G}} q^{\mathscr{L}}=\prod_{n=1}^{\infty}\left(1-q^{n}\right)^{-8} \times \prod_{r=\frac{1}{2}}^{\infty}\left(1+q^{r}\right)^{8}
$$

In order to compute the left-hand side of (6.5) we use the conjugation given in the second appendix. Once again the contribution now to the right-hand side of (6.5) coming from the $\left\{a_{n}^{\mu}, b_{n}, c_{n}\right\}$ oscillators is $\prod_{n=1}^{\infty}\left(1-q^{n}\right)^{-8}$. The contribution
from the Neveu-Schwarz oscillators is

$$
\begin{aligned}
\operatorname{Tr}_{\mathscr{H}(b)} \mathscr{C} q^{\mathscr{L}(b)} & =\prod_{\mu=0}^{9}{ }_{r=\frac{1}{2}}^{\infty} \operatorname{Tr}_{A_{r}^{\mu}} \mathscr{C} q^{r b_{r}^{\mu \dagger} b_{r}^{\mu}} \\
& =\prod_{\mu=0}^{9} \prod_{r=\frac{1}{2}}^{\infty}\left(1+(-1)^{\delta_{\mu, 0}} q^{r}\right)=\prod_{r=\frac{1}{2}}^{\infty}\left(1-q^{r}\right) \cdot\left(1+q^{r}\right)^{9} .
\end{aligned}
$$

Finally we compute the contribution coming from the superghosts. Notice that because of the nature of the conjugation $\mathscr{C}$ we only pick a contribution to the trace from states whose $\beta$ and $\gamma$ occupation numbers coincide. Therefore

$$
\begin{aligned}
\operatorname{Tr}_{\mathscr{H}}(\beta, \gamma) \mathscr{C} q^{\mathscr{L}^{(\beta, \gamma)}} & =\prod_{r=\frac{1}{2}}^{\infty} \operatorname{Tr}_{S_{r}} \mathscr{C} q^{r\left(N_{\beta}+N_{\gamma}\right)} \\
& =\prod_{r=\frac{1}{2}}^{\infty} \sum_{n=0}^{\infty} q^{2 r n}=\prod_{r=\frac{1}{2}}^{\infty}\left(1-q^{2 r}\right)^{-1}
\end{aligned}
$$


Combining all results we find

$$
\operatorname{Tr}_{C_{\infty}} \mathscr{C} q^{\mathscr{L}}=\prod_{n=1}^{\infty}\left(1-q^{n}\right)^{-8} \times \prod_{r=\frac{1}{2}}^{\infty}\left(1+q^{r}\right)^{8}
$$

which agrees with (6.14), hence proving the "no-ghost" theorem for the NeveuSchwarz sector.

\section{The Ramond Sector}

We first prove the "no-ghost" theorem for the Henneaux representation. We will then infer a similar result for the Schrödinger representation.

The relative subcomplex $C_{\infty}\left(\mathscr{R}, \mathscr{R}_{0} ; \mathscr{H}\right)$, which we abbreviate to $C_{\infty}$, has the following structure

$$
C_{\infty}=\mathscr{H}^{(a)} \otimes \mathscr{H}^{(b, c)} \otimes \mathscr{H}^{(d)} \otimes \mathscr{H}^{(\beta, \gamma)}
$$

where $\mathscr{H}^{(a)}$ and $\mathscr{H}^{(b, c)}$ were discussed already at the beginning of this section. As for the rest

$$
\begin{gathered}
\mathscr{H}^{(d)}=\bigotimes_{\mu=0}^{9} \bigotimes_{n=1}^{\infty} A_{n}^{\mu}, \\
\mathscr{H}^{(\beta, \gamma)}=\bigoplus_{n=1}^{\infty} S_{n} .
\end{gathered}
$$

Here $A_{n}^{\mu}$ is the Hilbert space of the $d_{n}^{\mu \dagger}$ oscillator and is isomorphic to the exterior algebra on one generator; and $S_{n}$ is the Hilbert space of the $\left\{\beta_{n}^{\dagger}, \gamma_{n}^{\dagger}\right\}$ oscillators and is isomorphic to the polynomial algebra in two variables.

Again the contribution to the right-hand side of (6.5) coming from the first two terms in the above decomposition is $\prod_{n=1}^{\infty}\left(1-q^{n}\right)^{-8}$. The Ramond oscillators
contribute

$$
\operatorname{Tr}_{\mathscr{H}(d)} q^{\mathscr{L}^{(d)}}=\prod_{\mu=0}^{9} \prod_{n=1}^{\infty} \operatorname{Tr}_{A_{n}^{\mu}} q^{n d_{n}^{\mu \dagger} d_{n}^{\mu}}=\prod_{n=1}^{\infty}\left(1+q^{n}\right)^{10}
$$

and the contribution from the superghosts is

$$
\begin{aligned}
\operatorname{Tr}_{\mathscr{H}^{(\beta, \epsilon)}(-1)^{\mathscr{G}} q^{\mathscr{L}^{(\beta, \gamma)}}}=\prod_{n=1}^{\infty} \operatorname{Tr}_{S_{n}}(-1)^{N_{\gamma}-N_{\beta}} q^{n\left(N_{\gamma}+N_{\beta}\right)} \\
=\prod_{n=1}^{\infty} \operatorname{Tr}_{S_{n}}\left(-q^{n}\right)^{N_{\gamma}+N_{\beta}}=\prod_{n=1}^{\infty} \sum_{m, p=0}^{\infty}\left(-q^{n}\right)^{m+p} \\
=\prod_{n=1}^{\infty}\left(\sum_{m=0}^{\infty}\left(-q^{n}\right)^{m}\right)^{2}=\prod_{n=1}^{\infty}\left(1+q^{n}\right)^{-2}
\end{aligned}
$$

where $N_{\beta}$ (respectively $N_{\gamma}$ ) is the number operator corresponding to the $\left\{\beta_{n}\right\}$ (respectively $\left\{\gamma_{n}\right\}$ ) oscillators. Putting everything together we find that

$$
\operatorname{Tr}_{C_{\infty}}(-1)^{\mathscr{G}} q^{\mathscr{L}}=\prod_{n=1}^{\infty}\left(\frac{1+q^{n}}{1-q^{n}}\right)^{8}
$$


In order to compute the left-hand side of (6.5) we use the conjugation given in the second appendix. The contribution coming from the $\left\{a_{n}^{\mu}, b_{n}, c_{n}\right\}$ oscillators is once again $\prod_{n=1}^{\infty}\left(1-q^{n}\right)^{-8}$. The Ramond oscillators contribute

$$
\begin{aligned}
\operatorname{Tr}_{\mathscr{H}^{(d)} \mathscr{C}} q^{\mathscr{L}^{(d)}} & =\prod_{\mu=0}^{9} \prod_{n=1}^{\infty} \operatorname{Tr}_{A_{n}^{\mu}} \mathscr{C} q^{n d_{n}^{\mu \dagger} d_{n}^{\mu}} \\
& =\prod_{\mu=0}^{9} \prod_{n=1}^{\infty}\left(1+(-1)^{\delta_{\mu, 0}} q^{n}\right)=\prod_{n=1}^{\infty}\left(1-q^{n}\right) \cdot\left(1+q^{n}\right)^{9} .
\end{aligned}
$$

Finally we compute the contribution coming from the superghosts. Just as in the Neveu-Schwarz sector we only pick a contribution to the trace from states whose $\beta$ and $\gamma$ occupation numbers coincide. Indeed,

Combining all results we find

$$
\begin{aligned}
\operatorname{Tr}_{\mathscr{H}^{(\beta, \gamma)}} \mathscr{C} q^{\mathscr{L}^{(\beta, \gamma)}} & =\prod_{n=1}^{\infty} \operatorname{Tr}_{S_{n}} \mathscr{C} q^{n\left(N_{\beta}+N_{\gamma}\right)} \\
& =\prod_{n=1}^{\infty} \sum_{m=0}^{\infty} q^{2 n m}=\prod_{n=1}^{\infty}\left(1-q^{2 n}\right)^{-1} .
\end{aligned}
$$

$$
\operatorname{Tr}_{C_{\infty}} \mathscr{C} q^{\mathscr{L}}=\prod_{n=1}^{\infty}\left(\frac{1+q^{n}}{1-q^{n}}\right)^{8},
$$

which agrees with (6.19), hence proving the "no-ghost" theorem.

In the Schrödinger representation $Q$ and $\mathbb{Q}$ are hermitian and therefore the inner product in cohomology does not depend on the particular cocycle chosen to represent a given class. Let $[\Psi]$ be a class in $H_{\infty}\left(\mathscr{R}, \mathscr{L}_{0} ; \mathscr{H}\right)$ and let $\psi \otimes 1$ denote a representative such that $T \psi=0$. Then $\psi$ defines a class in $H_{\infty}\left(\mathscr{R}, \mathscr{R}_{0} ; \mathscr{H}\right)$ in the Henneaux representation. We can normalize the inner product in the space of the superconformal ghosts' zero modes in such a way that the norm of $\psi \otimes 1$ agrees with the norm of $\psi$. Because $Q$ is hermitian, the norm of a class in $H_{\infty}\left(\mathscr{R}, \mathscr{R}_{0} ; \mathscr{H}\right)$ is independent of the representative; therefore the norm of $\psi$ is the norm of the class $[\psi]$ it represents. But by the "no-ghost" theorem just proven, the norm of $\psi$ is positive. Therefore the norm of $[\Psi]$ is positive. This proves the "no-ghost" theorem for the Schrödinger representation.

Finally we remark that the GSO projected NSR string is also free of ghosts. This is true because modular invariance also forces the GSO projection on the superghost spectrum which goes hand in hand with the GSO projection in the spectrum of the Neveu-Schwarz and Ramond oscillators. We leave the details of this calculation as an exercise.

\section{Appendix A. Computation of $H_{\infty}\left(\mathscr{S}_{-} ; \mathscr{M}\right)$}

In this section, we show that $H_{\infty}^{m}\left(\mathscr{S}_{-} ; \mathscr{M}\right)$ vanishes unless $m=0$, where $\mathscr{S}_{-}$is either the $\mathscr{N} \mathscr{S}_{-}$or $\mathscr{R}_{-}$superalgebras and $\mathscr{M}$ a Verma module of the respective algebra. ${ }^{12}$

\footnotetext{
${ }^{12}$ In actuality, this theorem holds for any superalgebra with values in a free module but we shall omit the general proof of this result so that the reader is not distracted by needless generalizations
} 
A lie superalgebra decomposes into odd and even subspaces so we can write $\mathscr{S}_{-}$ $=\mathscr{S}_{-}^{\text {even }} \oplus \mathscr{S}_{-}^{\text {odd }}$. Let us choose a basis in each subspace and denote them by $\left\{e_{i}\right\}$ and $\left\{f_{\alpha}\right\}$, respectively. A basis for the Verma module, $\mathscr{M}$, is then given by the highest weight vector together with the monomials

$$
\left\{e_{i_{1}} e_{i_{2}} \cdots e_{i_{n}} f_{\alpha_{1}} f_{\alpha_{2}} \cdots f_{\alpha_{m}}\right\} \text {, }
$$

where all of the subscripts are integers satisfying $i_{1} \leqq i_{2} \cdots \leqq i_{n}$ and $\alpha_{1}<\alpha_{2} \cdots<\alpha_{m}$ for some positive integers $m$ and $n$. Notice that we have omitted writing the highest weight vector explicitly in order to simplify the notation. A basis for the cochains $C_{\infty}\left(\mathscr{S}_{-} ; \mathscr{M}\right)$ is given by

where

$$
\left\{\beta_{\alpha_{1}}^{\dagger} \beta_{\alpha_{2}}^{\dagger} \cdots \beta_{\alpha_{k}}^{\dagger} b_{i_{1}}^{\dagger} b_{i_{2}}^{\dagger} \cdots b_{i_{1}}^{\dagger} \otimes e_{j_{1}} e_{j_{2}} \cdots e_{j_{m}} f_{\lambda_{1}} f_{\lambda_{2}} \cdots f_{\lambda_{n}}\right\}
$$

$$
\begin{gathered}
i_{1}<i_{2}<\cdots<i_{l}, \quad \text { and } \quad \alpha_{1} \leqq \alpha_{2} \cdots \leqq \alpha_{k}, \\
j_{1} \leqq j_{2} \leqq \cdots \leqq j_{m}, \quad \text { and } \quad \lambda_{1}<\lambda_{2}<\cdots<\lambda_{n} .
\end{gathered}
$$

It is understood that the antighosts are acting upon the usual ghost vacuum. One might have expected to see some $c$ 's and $\gamma$ 's in the above expression but we recall that $\mathscr{S}_{-}$is only "half" of the full superalgebra and the corresponding $c$ 's and $\gamma$ 's to this part of the superalgebra are the annihilation operators in those oscillators. Therefore, there are no such terms in $\mathscr{C}_{\infty}\left(\mathscr{S}_{-} ; \mathscr{M}\right)$.

Having characterized the cochains, we proceed to construct a spectral sequence which converges to $H_{\infty}\left(\mathscr{S}_{-} ; \mathscr{M}\right)$. Let's define

$$
\Omega=\beta_{\alpha_{1}}^{\dagger} \cdots \beta_{\alpha_{K}}^{\dagger} b_{i_{1}}^{\dagger} \cdots b_{i_{L}}^{\dagger} \otimes e_{j_{1}} \cdots e_{j_{M}} f_{\lambda_{1}} \cdots f_{\lambda_{N}} .
$$

We then define a filtration degree on $\mathscr{C}_{\infty}\left(\mathscr{S}_{-} ; \mathscr{M}\right)$ via

$$
\operatorname{fdeg} \Omega=K+L+M+N \text {. }
$$

This gives us a filtration of $\mathscr{C}_{\infty}\left(\mathscr{S}_{-} ; \mathscr{M}\right)$

$$
F^{p} \mathscr{C}_{\infty}\left(\mathscr{S}_{-} ; \mathscr{M}\right)=\left\{\omega \in \mathscr{C}_{\infty}\left(\mathscr{S}_{-} ; \mathscr{M}\right) \mid \mathrm{fdeg} \omega \leqq p\right\}
$$

In the case of the Neveu-Schwarz algebra, this is a half-integral filtration while in the case the Ramond algebra this is an integral filtration. We shall proceed as if this filtration were integral throughout the remainder of this section in order to avoid unnecessary clutter. The arguments for the case of the half-integral filtration are exactly the same.

It is quite easy to see that $F \mathscr{C}_{\infty}\left(\mathscr{S}_{-} ; \mathscr{M}\right)$ is a filtration since it satisfies

$$
F^{p} \mathscr{C}_{\infty}\left(\mathscr{S}_{-} ; \mathscr{M}\right) \subseteq F^{p+1} \mathscr{C}_{\infty}\left(\mathscr{S}_{-} ; \mathscr{M}\right) \quad \forall p,
$$

and all of the terms in the coboundary operator, $d$, have filtration degrees that are nonpositive. Furthermore, we observe that $L_{0}$ is diagonalizable on $\mathscr{C}_{\infty}\left(\mathscr{S}_{-} ; \mathscr{M}\right)$ and commutes with $d$. Let us denote a subspace of $\mathscr{C}_{\infty}\left(\mathscr{S}_{-} ; \mathscr{M}\right)$ with $L_{0}$ eigenvalue $\mu$ by $\mathscr{C}_{\infty}\left(\mathscr{S}_{-} ; \mathscr{M}\right)^{\mu}$ so that

$$
\mathscr{C}_{\infty}\left(\mathscr{S}_{-} ; \mathscr{M}\right)=\bigoplus_{\mu} \mathscr{C}_{\infty}\left(\mathscr{S}_{-} ; \mathscr{M}\right)^{\mu}
$$


then their associated cohomologies break up into

$$
H_{\infty}\left(\mathscr{S}_{-} ; \mathscr{M}\right)=\bigoplus_{\mu} H_{\infty}\left(\mathscr{S}_{-} ; \mathscr{M}\right)^{\mu}
$$

This decomposition is interesting because the dimension of $\mathscr{C}_{\infty}\left(\mathscr{S}_{-} ; \mathscr{M}\right)^{\mu}$ is finite at every value of $\mu$. This implies that fdeg is bounded. Hence, the above filtration when restricted to a particular $L_{0}$ value, $\mu$, forms a bounded filtration. Therefore, there exists a spectral sequence which converges finitely to $H_{\infty}\left(\mathscr{S}_{-} ; \mathscr{M}\right)^{\mu}$. Suppose we are able to show that $H_{\infty}^{m}\left(\mathscr{S}_{-} ; \mathscr{M}\right)^{\mu}=0$ for all $\mu$ and $m \neq 0$ then it is certainly true that $H_{\infty}^{m}\left(\mathscr{S}_{-} ; \mathscr{M}\right)=0$ unless $m \neq 0$. We will now show that this is, indeed, the case. Unless otherwise mentioned, it will be understood that we are restricting ourselves to cochains and cohomologies at a particular value of $\mu$ and shall hitherto drop all references to $\mu$.

Let us compute the $E_{1}$ term in the spectral sequence. Recall that $E_{1}=H\left(\operatorname{Gr} \mathscr{C}_{\infty}\left(\mathscr{S}_{-} ; \mathscr{M}\right)\right)$ where $\operatorname{Gr} \mathscr{C}_{\infty}\left(\mathscr{S}_{-} ; \mathscr{M}\right)$ carries the differential induced by $d$ on $F \mathscr{C}_{\infty}\left(\mathscr{S}_{-} ; \mathscr{M}\right)$. The only terms in $d$ which have fdeg $=0$ are

$$
d=\sum_{i} c_{i} \otimes e_{i}+\sum_{\alpha} \gamma_{\alpha} \otimes f_{\alpha}
$$

These are the only terms contributing to action of the induced differential on $\operatorname{Gr} \mathscr{C}_{\infty}\left(\mathscr{S}_{-} ; \mathscr{M}\right)$. Mor explicitly, we can write

$$
\begin{aligned}
d \Omega= & \sum_{i} \beta_{\alpha_{1}}^{\dagger} \cdots \beta_{\alpha_{K}}^{\dagger} c_{i} b_{i_{1}}^{\dagger} \cdots b_{i_{L}}^{\dagger} \otimes e_{i} e_{j_{1}} \cdots e_{j_{M}} f_{\lambda_{1}} \cdots f_{\lambda_{N}} \\
& +(-1)^{L} \sum_{\alpha} \gamma_{\alpha} \beta_{\alpha_{1}}^{\dagger} \cdots \beta_{\alpha_{K}}^{\dagger} b_{i_{1}}^{\dagger} \cdots b_{i_{L}}^{\dagger} \otimes e_{j_{1}} \cdots e_{j_{M}} f_{\alpha} f_{\lambda_{1}} \cdots f_{\lambda_{N}}
\end{aligned}
$$

Two remarks are in order. First of all notice that the above sums are actually finite and second that the terms above are to be taken modulo $F^{p-1} \mathscr{C}_{\infty}\left(\mathscr{S}_{-} ; \mathscr{M}\right)$. Now define a linear map $\Gamma: \operatorname{Gr} \mathscr{C}_{\infty}^{m}\left(\mathscr{S}_{-} ; \mathscr{M}\right) \rightarrow \operatorname{Gr} \mathscr{C}_{\infty}^{m-1}\left(\mathscr{S}_{-} ; \mathscr{M}\right)$ for all $m>0$ by

$$
\begin{aligned}
\Gamma \omega= & \sum_{l=1}^{N} \beta_{\alpha_{1}}^{\dagger} \cdots \beta_{\alpha_{K}}^{\dagger} b_{j_{l}}^{\dagger} b_{i_{1}}^{\dagger} \cdots b_{i_{L}}^{\dagger} \otimes e_{j_{1}} \cdots \widehat{e_{j_{l}}} \cdots e_{j_{M}} f_{\lambda_{1}} \cdots f_{\lambda_{N}} \\
& +\sum_{l=1}^{N}(-1)^{L+l-1} \beta_{\lambda_{l}}^{\dagger} \beta_{\alpha_{1}}^{\dagger} \cdots \beta_{\alpha_{K}}^{\dagger} b_{i_{1}}^{\dagger} \cdots b_{i_{L}} \otimes e_{j_{1}} \cdots e_{j_{M}} f_{\lambda_{1}} \cdots \widehat{f_{\lambda_{N}}},
\end{aligned}
$$

where an element with a over it means that it is missing from that term. A calculation shows that this map satisfies the relation

$$
(d \Gamma+\Gamma d) \Omega=(K+L+M+N) \Omega .
$$

So, consider any $\Omega \in \mathscr{C}_{\infty}^{m}\left(\mathscr{S}_{-} ; \mathscr{M}\right)$ where $m>0$ and $d \Omega=0$ then the previous equation implies that

$$
d \Gamma \Omega=(K+L+M+N) \Omega .
$$

Since $m>0$, this means that $K+L+M+N \neq 0$ which implies that

$$
H^{m}\left(\operatorname{Gr} \mathscr{C}_{\infty}\left(\mathscr{S}_{-} ; \mathscr{M}\right)\right)=0 \quad \text { if } \quad m \neq 0
$$


Because we have the convergence of the spectral sequence $\left(E_{r}^{m}\right) \Rightarrow H^{m}$ we are able to conclude that

$$
H^{m}\left(\mathscr{C}_{\infty}\left(\mathscr{S}_{-} ; \mathscr{M}\right)\right)=0 \quad \text { if } \quad m \neq 0
$$

\section{Appendix B. A Positive-Definite Inner Product for the NSR Fock Space}

In [4] we proved a Poincare duality theorem which requires two things: first that the Fock space decomposes into a direct sum of finite dimensional subspace which are stabilized by the BRST operator and second that there exists a positive definite inner product in the Fock space. The first point is obvious since there are only a finite number of states of a given level. We address the second question in this appendix, where we construct a positive-definite inner product explicitly. The inner product is defined from the original one imposed by the quantization procedure by the introduction of a self-adjoint involution $\mathscr{C}$ in such a way that the new inner product is

$$
\langle\psi, \phi\rangle \hat{c}=\langle\psi, \mathscr{C} \phi\rangle,
$$

where $\langle$,$\rangle is the original inner product and \psi$ and $\phi$ are vectors in the Fock space. On the ghost and anti-ghost oscillators this conjugation $\mathscr{C}$ plays the rôle of the SerreHodge $\bar{\star}$ operator in complex geometry [13] and therefore is consistent with the "semi-infinite" form interpretation of the ghost Fock space.

First a word of caution. Our ghost oscillators are not the natural ones but are unitarily related to them. In our conventions the mode expansion of the conformal ghost and antighost fields at $\tau=0$ are the following:

$$
\begin{aligned}
& b(\sigma)=b_{0}+\sum_{m>0} \sqrt{m}\left(b_{m} e^{i m \sigma}+b_{-m} e^{-i m \sigma}\right), \\
& c(\sigma)=c_{0}+\sum_{m>0} \frac{1}{\sqrt{m}}\left(c_{m} e^{i m \sigma}+c_{-m} e^{-i m \sigma}\right),
\end{aligned}
$$

and similarly for the superconformal ghosts. This seemingly unnatural choice of mode expansion turns out to be the natural one in our context. It will allow us to identify the involution $\mathscr{C}$ above with ghost conjugation when acting on ghosts and antighosts.

For the $\left\{a_{n}^{\mu}, b_{n}, c_{n}\right\}$ oscillators we define $\mathscr{C}$ as follows

$$
\begin{aligned}
& \mathscr{C} p^{\mu} \mathscr{C}=p^{\mu}, \\
& \mathscr{C} a_{n}^{0} \mathscr{C}=-a_{n}^{0}, \quad \mathscr{C} a_{n}^{i} \mathscr{C}=a_{n}^{i}, \quad \forall i=1 \ldots 9 \quad \text { and } \forall n \neq 0, \\
& \mathscr{C} c_{n} \mathscr{C}=b_{n}, \quad \mathscr{C} b_{n} \mathscr{C}=c_{n}, \quad(\forall n \in Z) .
\end{aligned}
$$

For the Neveu-Schwarz oscillators the conjugation with the desired properties turns out to be the following

$$
\begin{gathered}
\mathscr{C} b_{s}^{\mu} \mathscr{C}=(-1)^{\delta_{\mu, 0}} b_{s}^{\mu} \quad \forall s \in \mathrm{Z}+\frac{1}{2}, \\
\mathscr{C} \gamma_{r} \mathscr{C}=\beta_{r}, \quad \mathscr{C} \beta_{r} \mathscr{C}=\gamma_{r}, \\
\mathscr{C} \gamma_{-r} \mathscr{C}=-\beta_{-r}, \quad \mathscr{C} \beta_{-r} \mathscr{C}=-\gamma_{-r} \quad \forall r \in \mathbf{N}-\frac{1}{2},
\end{gathered}
$$


and for the Ramond oscillators it is very similar:

$$
\begin{gathered}
\mathscr{C} d_{m}^{\mu} \mathscr{C}=(-1)^{\delta_{\mu}, 0} d_{m}^{\mu} \quad \forall m \in \mathbf{Z} \\
\mathscr{C} \gamma_{n} \mathscr{C}=\beta_{n}, \quad \mathscr{C} \beta_{n} \mathscr{C}=\gamma_{n}, \\
\mathscr{C} \gamma_{-n} \mathscr{C}=-\beta_{-n} \quad \mathscr{C} \beta_{-n} \mathscr{C}=-\gamma_{-n} \quad \forall n \in \mathbf{N} .
\end{gathered}
$$

For the ghost zero modes $\left\{\beta_{0}, \gamma_{0}\right\}$ there are two possibilities depending on the choice of Hilbert space that we choose for their representation. As discussed in Sect. 2 we can choose the Hilbert space in which they are self-adjoint in which case we already have a positive definite inner product and therefore $\mathscr{C}$ acts leaves them inert. On the other hand, following Henneaux [12], we can treat them as annihilation and creation operators, in which case $\beta_{0}$ and $\gamma_{0}$ are mutual adjoints. It is interesting to remark that in this case there is no self-adjoint involution $\mathscr{C}$ which yields this adjointness property from the original ones for $\beta_{0}$ and $\gamma_{0}$. However these are the only operators acting in this space and hence there is no need - in order to compute adjoints - for the operator $\mathscr{C}$ itself to exist.

To show that the new inner product defined by (B.1) is indeed positive-definite is completely straightforward and is left as an exercise for the reader.

Acknowledgements. We would like to thank Martin Roček for suggesting the problem. One of us (JMF) would like to thank Claude LeBrun for some valuable insight into spectral sequences. We would also like to acknowledge conversations with Francisco Figueirido, Jim McCarthy, and Eduardo Ramos.

\section{References}

1. Frenkel, I.B., Garland, H., Zuckerman, G.J.: Proc. Natl. Acad. Sci. USA 83, 8442 (1986)

2. Fuks, D.B.: Cohomology of infinite-dimensional Lie algebras. New York: Plenum Press 1986

3. Spiegelglas, M.: Nucl. Phys. B283 205 (1987)

4. Figueroa-O'Farrill, J.M., Kimura, T.: Stony Brook Preprint ITP-SB-88-34 (revised)

5. Lang, S: Algebra. Reading, MA: Addison-Wesley 1984

6. Griffiths, P., Harris, J.: Principles of algebraic geometry. New York: Wiley 1978

7. Bott, R., Tu, L.: Differential forms in algebraic topology. Berlin, Heidelberg, New York: Springer 1982

8. Hilton, P.J., Stammbach, U.: A course in homological algebra. Berlin, Heidelberg, New York: Springer 1970

9. MacLane, S.: Homology. New York: Academic Press 1963

10. Green, M., Schwarz, J.H., Witten, E.: Superstring theory. Cambridge: Cambridge Univ. Press 1987

11. Brower, R.C., Friedman, K.: Phys. Rev. D7, 535 (1972)

12. Henneaux, M.: Phys. Lett. 183B, 59 (1987)

13. Figueroa-O'Farrill, J.M., Kimura, T.: Stony Brook Preprint ITP-SB-88-35 (revised)

14. Koszul, J.-L.: Bull. Soc. Mat. France 78, 65 (1950)

Communicated by L. Alvarez-Gaumé

Received October 4, 1988 